\title{
Composing and Decomposing Op-Based CRDTs with Semidirect Products
}

\author{
MATTHEW WEIDNER, Carnegie Mellon University, USA \\ HEATHER MILLER, Carnegie Mellon University, USA \\ CHRISTOPHER MEIKLEJOHN, Carnegie Mellon University, USA
}

\begin{abstract}
Operation-based Conflict-free Replicated Data Types (CRDTs) are eventually consistent replicated data types that automatically resolve conflicts between concurrent operations. Op-based CRDTs must be designed differently for each data type, and current designs use ad-hoc techniques to handle concurrent operations that do not naturally commute. We present a new construction, the semidirect product of op-based CRDTs, which combines the operations of two CRDTs into one while handling conflicts between their concurrent operations in a uniform way. We demonstrate the construction's utility by using it to construct novel CRDTs, as well as decomposing several existing CRDTs as semidirect products of simpler CRDTs. Although it reproduces common CRDT semantics, the semidirect product can be viewed as a restricted kind of operational transformation, thus forming a bridge between these two opposing techniques for constructing replicated data types.
\end{abstract}

CCS Concepts: • Software and its engineering $\rightarrow$ Data types and structures; • Theory of computation $\rightarrow$ Distributed algorithms.

Additional Key Words and Phrases: CRDTs, Operational Transformation, Eventual Consistency

ACM Reference Format:

Matthew Weidner, Heather Miller, and Christopher Meiklejohn. 2020. Composing and Decomposing Op-Based CRDTs with Semidirect Products. Proc. ACM Program. Lang. 4, ICFP, Article 94 (August 2020), 27 pages. https://doi.org/10.1145/3408976

\section{INTRODUCTION}

Geo-replication of data is a technique used in many distributed applications, such as distributed databases and collaborative document editing programs, to reduce user-perceived latency and increase fault tolerance. However, geo-replication is challenging: in order to achieve fault tolerance and high performance, replicas need to avoid the large communication costs of coordinating over geographically distributed networks. This is typically achieved by allowing users to update their own replicas locally, then asynchronously propagate updates to other replicas in the background. This poses an interesting concurrency challenge: what should the final outcome be in the face of conflicting concurrent operations by different replicas, once all of the updates are delivered to all replicas?

Conflict-free Replicated Data Types (CRDTs) [Preguiça et al. 2018; Shapiro et al. 2011] are a class of highly available replicated data types that provide a principled solution to this problem. They allow developers to write applications using ordinary sequential data type operations acting on

Authors' addresses: Matthew Weidner, maweidne@andrew.cmu.edu, Carnegie Mellon University, 5000 Forbes Avenue, Pittsburgh, PA, 15213, USA; Heather Miller, heather.miller@cs.cmu.edu, Carnegie Mellon University, 5000 Forbes Avenue, Pittsburgh, PA, 15213, USA; Christopher Meiklejohn, cmeiklej@andrew.cmu.edu, Carnegie Mellon University, 5000 Forbes Avenue, Pittsburgh, PA, 15213, USA.

This work is licensed under a Creative Commons Attribution 4.0 International License.

(C) 2020 Copyright held by the owner/author(s).

2475-1421/2020/8-ART94

https://doi.org/10.1145/3408976

Proc. ACM Program. Lang., Vol. 4, No. ICFP, Article 94. Publication date: August 2020. 
replicated state. Updates to the replicated state are propagated asynchronously, with conflicts between concurrent operations resolved automatically using type-specific rules. CRDTs have been deployed in highly available geo-replicated data storage systems such as Antidote [AntidoteDB 2019] and Riak [Basho 2015].

Operation-based (op-based) CRDTs [Preguiça et al. 2018; Shapiro et al. 2011] are a type of CRDT that function by converting sequential data type operations into messages, which are then broadcast to all replicas in causal order, a natural partial order that can be enforced without coordination. Replicas apply received messages to their state in such a way that they all end up in the same state, even if they receive concurrent messages in different orders. A simple example is a counter supporting increment and decrement operations: these operations naturally commute, so they can be directly broadcast to all replicas, which then apply the operations in the order they receive them. More generally, any commutative data type is trivially an op-based CRDT.

However, op-based CRDTs for non-commutative data types must explicitly handle conflicts between non-commuting concurrent messages. For example, a set CRDT must decide what to do in the face of operations that concurrently add and remove an element to the set, while ensuring that all replicas eventually reach the same result. Current designs handle such conflicts using ad-hoc techniques that differ for each data type. It is thus difficult to design CRDTs for new data types or to add operations to existing CRDTs.

For example, suppose we are making a Slack-like chat application containing multiple "channels" that users can join. We can store the mapping from channel names to the set of users in each channel using a CRDT dictionary [AntidoteDB 2019; Basho 2015], with CRDT sets as the values, as shown in Figure 1. In this example, there are two replicas, replica $A$ and replica $B$, stored at two different nodes, $A$ and $B$, that are executing the following application code.

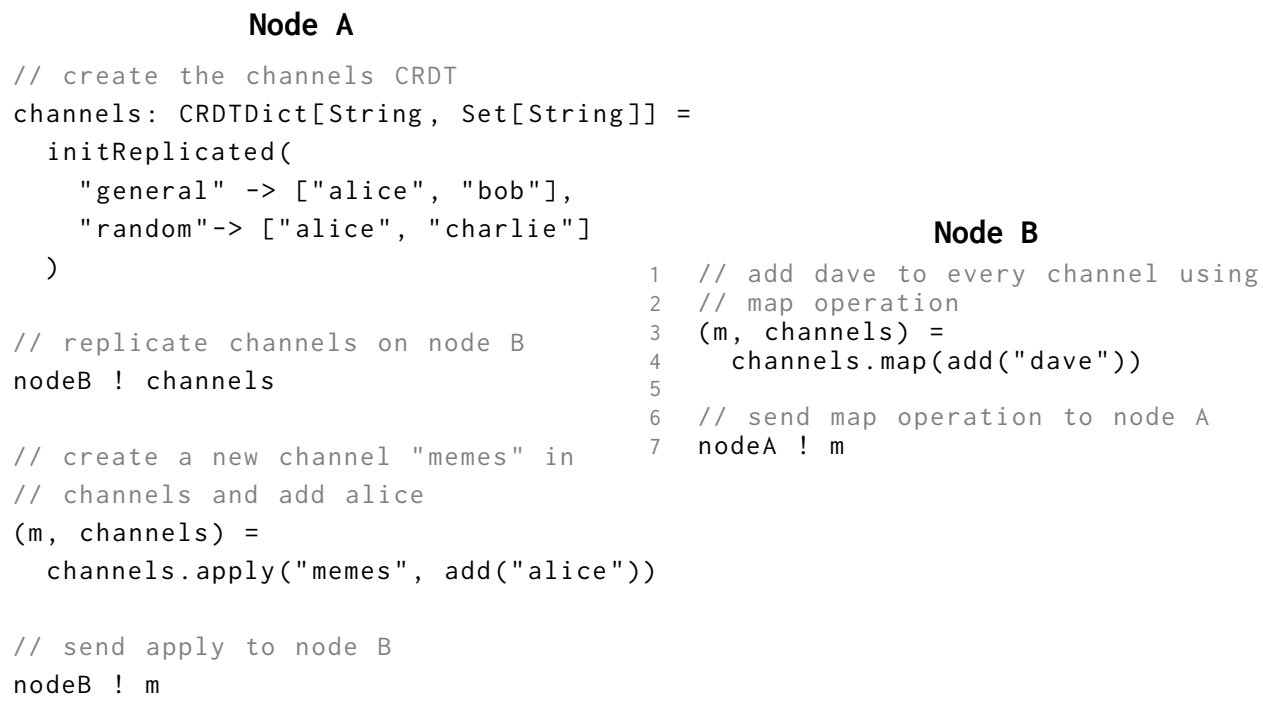

ig. 1. Example program showing distributed use of the replicated value channels in a Slack-like chat application by two nodes, $A$ and $B$. apply $(k$, add $(v))$ adds $v$ to the set at key $k$, initializing the value at $k$ if necessary, while map $(\operatorname{add}(v))$ adds $v$ to every set in the dictionary. Unfortunately, these two operations do not commute, so after receiving each other's messages, $A$ and $B$ end up with different values for channels (see Figure 2). 

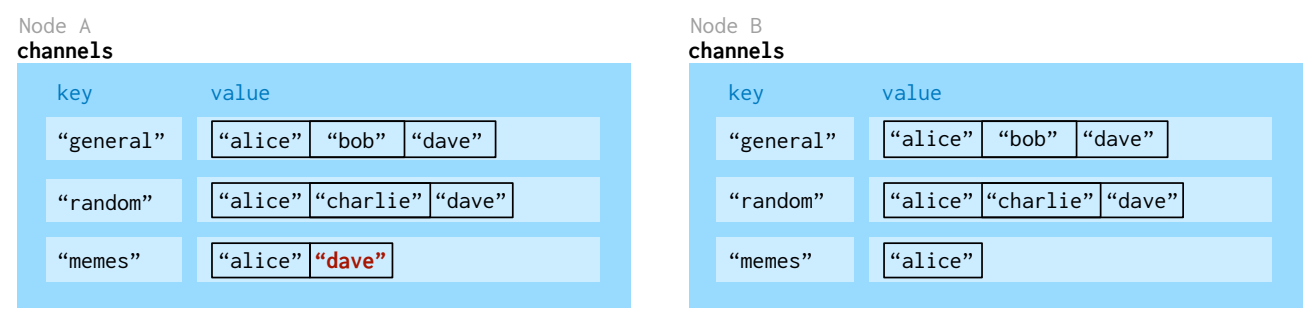

Fig. 2. Replicas $A$ and $B$ after executing the example program shown in Figure 1. Because the operations from Figure 1 do not commute, $A$ and $B$ end up with different values for channels.

Suppose we wish to add a map operation map, which applies a function to every value currently present in the dictionary. This operation could be used to add a user dave to every channel at node $B$. In Figure 1, we run into the issue that this operation does not commute with operations that initialize the value at a key: node $B$ processes the map operation before the operation that initializes channel memes, so it does not add dave to memes, while node $A$ processes the operations in the opposite order, so it does add dave to memes, as shown in Figure 2. Hence it is not obvious how to add such an operation to a dictionary CRDT, and as a result, existing dictionary designs do not include one.

Our goal in this paper is to make it easy to add operations like this to CRDTs, even when they do not commute with existing operations. More generally, we aim to compose op-based CRDTs, combining their sets of operations while handling conflicts between them in a uniform way. One can then add operations to an existing CRDT by composing it with another CRDT that only implements the new operations.

We do so by presenting a new CRDT construction technique, the semidirect product of op-based CRDTs, which composes two op-based CRDTs into a new CRDT with both of their operations. We demonstrate through numerous examples that the semidirect product can be used both to create novel CRDTs through composition and to decompose existing CRDTs into simpler components. Our novel CRDT designs include a dictionary with map operations, sequence types with reverse or range removal operations, and an integer register supporting addition and multiplication operations. These examples widen the range of data types available as CRDTs without substantial design effort. The existing CRDTs we decompose include sets, flags, and resettable CRDTs. These examples help explain seemingly ad-hoc CRDT designs by decomposing them into simpler parts, many of which are commutative data types, with the semidirect product handling conflicts between non-commuting concurrent operations in a uniform way.

We further demonstrate the semidirect product's generality by giving a criterion for when an op-based CRDT designed using an existing general model [Baquero et al. 2017] can be decomposed as a semidirect product of simpler CRDTs, and we show that it applies in many cases.

Briefly, the semidirect product works as follows. Conflicts between non-commuting concurrent operations from the two CRDTs are handled according to an arbitration order, which specifies that operations from the first CRDT should be applied before concurrent operations from the second CRDT. Since directly reordering operations in this way is not always defined, we instead make use of a transformation function, which transforms operations from the first CRDT to take into account concurrent operations from the second CRDT. Our construction can thus be viewed as a restricted kind of operational transformation [Ressel et al. 1996], an alternative method of constructing replicated data types that is often contrasted with CRDTs, as we discuss in Section 


$\begin{array}{cll}\Sigma & : & \text { Set of states } \\ \operatorname{prepare}(o, \sigma, r): & : \begin{array}{l}\text { Prepares a message } m \text { given an op- } \\ \text { eration } o \text { by replica } r \text { in state } \sigma\end{array} \\ \operatorname{effect}(m, \sigma): & : \begin{array}{l}\text { Partial function that applies a pre- } \\ \text { pared message } m \text { to a state } \sigma, \text { re- }\end{array} \\ & \text { turning the resulting state } \\ m \cdot \sigma \quad: & \begin{array}{l}\text { Abbreviation for effect }(m, \sigma) \text { when } \\ \text { the CRDT is clear from context }\end{array} \\ \operatorname{eval}(q, \sigma) \quad: \begin{array}{l}\text { Read-only evaluation of a query } q \\ \text { on a state } \sigma\end{array}\end{array}$

Fig. 3. Components of an op-based CRDT.

6.1. In comparison with general operational transformation, the semidirect product has reduced complexity, thus avoiding CRDT proponents' main criticism of operational transformation.

The semidirect product of CRDTs is named after the semidirect product of groups, a construction from abstract algebra that inspired their design, as described in the appendix.

A summary of this paper appeared at the PaPoC Workshop 2020 [Weidner et al. 2020].

\section{BACKGROUND ON OP-BASED CRDTS}

Notation. We use $f: S \rightarrow T$ to denote that $f$ is a partial function from $S$ to $T$, i.e., a function defined on a subset of $S$. We write $f(s)=\perp$ to indicate that $f$ is not defined on $s$.

As mentioned above, an op-based CRDT is a replicated data type in which replicas convert sequential data type operations into messages that they broadcast to other replicas. Replicas receive these messages and apply them to their states in causal order (defined below), in such a way that concurrent messages commute.

Definition 2.1. The causal order is the partial order $<$ on messages defined by the transitive closure of the rule: $m_{1} \prec m_{2}$ if the replica that sent $m_{2}$ did so after receiving $m_{1}$, or if $m_{1}$ and $m_{2}$ were sent by the same replica and $m_{1}$ was sent before $m_{2}$. Two messages $m_{1}, m_{2}$ are concurrent if $m_{1} \nless m_{2}$ and $m_{2} \nless m_{1}$. The requirement that replicas receive messages in causal order means that a replica should not receive a message $m_{2}$ until after it has received all messages $m_{1} \prec m_{2}$.

A simple example is the op-based counter CRDT. This has state space $\mathbb{Z}$ with operations add $(n)$ for $n \in \mathbb{Z}$, acting as $\operatorname{add}(n): \sigma \mapsto n+\sigma$. These operations naturally commute.

More complicated CRDTs, such as set CRDTs, attach extra metadata to states, to handle the fact that their operations do not naturally commute. Additionally, instead of sending operations directly in their messages to other replicas, they may modify them or attach metadata.

Formally, we adopt the following definition of an op-based CRDT, based on that of Shapiro et al. [2011] but with notation more similar to Baquero et al. [2017, §3].

Definition 2.2. An op-based $C R D T$ is a tuple $\left(\Sigma, \sigma^{0}\right.$, prepare, effect, eval) of the form given in Figure 3, such that:

(i) For all $m=$ prepare $(o, \sigma, r), m \cdot \sigma \neq \perp$

(ii) For all $\sigma \in \sum$ and all messages $m_{1}, m_{2}$, if $m_{1}$ and $m_{2}$ can be sent concurrently in an execution of the CRDT (formalized in Algorithm 1 below), and $m_{1} \cdot \sigma \neq \perp$ and $m_{2} \cdot \sigma \neq \perp$, then

$$
m_{1} \cdot\left(m_{2} \cdot \sigma\right)=m_{2} \cdot\left(m_{1} \cdot \sigma\right) \neq \perp \text {. }
$$

The definition ensures that operations commute if they could be issued concurrently. 
Example 2.3. We can formalize the op-based counter CRDT as $\Sigma=\mathbb{Z}$, prepare $(\operatorname{add}(n), \sigma, r)=$ $\operatorname{add}(n), \operatorname{effect}(\operatorname{add}(n), \sigma)=n+\sigma$, and eval $($ value, $\sigma)=\sigma$.

Algorithm 1 formalizes the use of an op-based CRDT by a group of replicas. Initially, all replicas are in the initial state $\sigma^{0}$. At any time, a replica can issue an operation $o$, causing a message $m$ to be prepared and broadcast to all replicas. All replicas apply received messages to their state in causal order using effect. Replicas can also be queried to return external information about their state. For instance, a set CRDT could have a query to return the elements of the set based on the internal metadata-enhanced state $\sigma$.

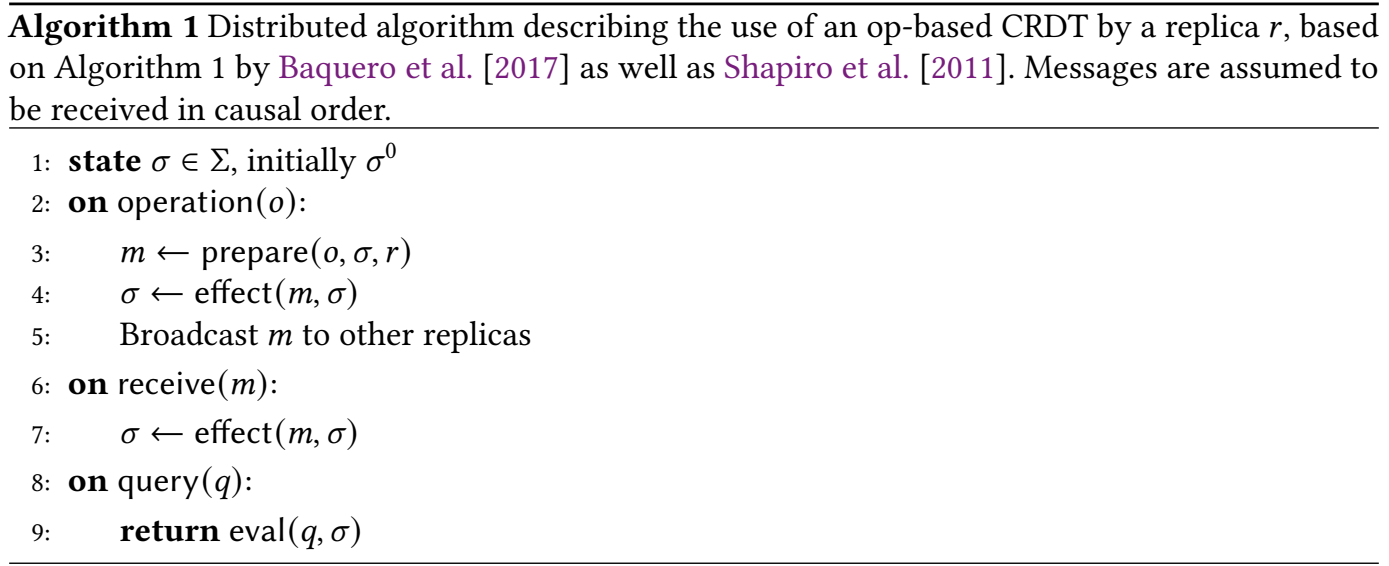

Properties (i) and (ii) of an op-based CRDT imply eventual consistency: two replicas that have received the same messages end up in the same state $\sigma \neq \perp$ when using Algorithm 1, even if they receive concurrent messages in different orders [Shapiro et al. 2011, Proposition 2.2].

The main advantage of op-based CRDTs over strongly consistent data types, like data in traditional databases, is that causally ordered message delivery can be enforced without coordination between replicas. Thus replicas can immediately apply changes to their own copies of the data, and they can send messages without requiring a costly consensus protocol to put all messages in a consistent total order [Défago et al. 2004].

Remark 2.4. One way to enforce causally ordered delivery in an implementation of Algorithm 1 is to use vector clocks [Fidge 1988; Mattern 1989], which are functions from replica ids to $\mathbb{N}$. Each replica maintains a vector clock $t$ such that $t(r)$ is the number of messages it has received from replica $r$. When sending a message $m$, a replica increments its own entry in $t$ and attaches a copy of $t$ to $m$ as metadata (its timestamp). Thus $m_{1} \leq m_{2}$ if and only if their corresponding timestamps $t_{1}, t_{2}$ satisfy $t_{1} \leq t_{2}$, and they are concurrent if and only if $t_{1} \not \leq t_{2}$ and $t_{2} \not \leq t_{1}$. To enforce causally ordered delivery, we wait to deliver a message $m$ from a replica $r_{1}$ with timestamp $t$ to another replica $r_{2}$ until $r_{2}$ 's vector clock $t_{2}$ satisfies: $t_{2}\left(r_{1}\right)=t\left(r_{1}\right)-1$ and for all $r \neq r_{1}, t_{2}(r) \geq t(r)$. Besides enforcing causally ordered delivery, some CRDT algorithms explicitly include vector clocks in messages so that effect can query the causal order. Our semidirect product construction does this to determine when messages are concurrent.

Remark 2.5. We allow effect to have undefined values only because this makes it easier to formally satisfy property (ii). Properties (i) and (ii) guarantee that in any execution of Algorithm 1, every replica's state will always be defined, hence undefined states will never appear in practice. For example, one could set effect $(m, \sigma)=\perp$ if $m$ and $\sigma$ include vector clocks and $m$ 's clock is not a 
direct successor to $\sigma$ 's clock. This scenario is impossible in practice due to causally ordered delivery, so there is no need to require that $m$ commutes with other messages applied to $\sigma$.

\section{SEMIDIRECT PRODUCTS}

\subsection{Motivating Example}

To motivate the semidirect product construction, suppose we wish to construct an integer register CRDT supporting addition and multiplication operations. Specifically, for $n \in \mathbb{Z}$, we want to allow operations $\operatorname{add}(n): \sigma \mapsto n+\sigma$ and $\operatorname{mult}(n): \sigma \mapsto n \times \sigma$. The add operations alone form a CRDT because they naturally commute, and likewise for the mult operations, but they do not commute with each other. Because of this, such a CRDT was not previously known, despite the simplicity of its interface.

As a first attempt, let us dictate that in the face of concurrent add and mult operations, a replica applies all of the add operations first, followed by all of the mult operations. For example, starting in state 1 , if two replicas concurrently issue operations add(1) and mult(3), then regardless of the order in which they receive these operations, all replicas compute the final state as mult $(3) \cdot(\operatorname{add}(1) \cdot 1)=6$. We call this order of operations the arbitration order. Non-concurrent operations should continue to be applied in the order they were generated, i.e., in causal order.

Unfortunately, this approach is not well-defined in general. For example, consider the following scenario:

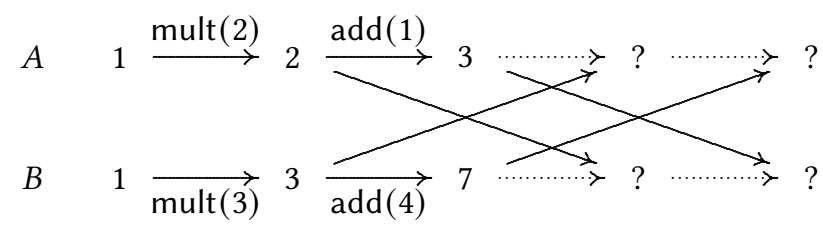

Here replicas $A$ and $B$ both start in state 1 . Replica $A$ issues operations mult(2) followed by add(1), while $B$ concurrently issues operations mult(3) followed by add(4). After receiving each others' messages, both replicas attempt to order these four messages according to the above rules. However, this creates a loop:

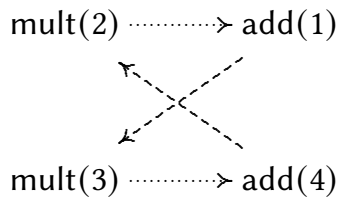

Indeed, the arbitration order (dashed arrows) dictates that each replica should apply add(1) before the concurrent operation mult(3), and add(4) before mult(2). Meanwhile, to respect the causal order (dotted arrows), replicas should apply mult(2) before add(1) and mult(3) before add(4). Thus there is no valid way to compute the current state.

As a second attempt, observe that applying add $(m)$ followed by mult $(n)$ is the same as applying $\operatorname{mult}(n)$ followed by add $(\mathrm{nm})$, by the distributive property. Thus in the face of concurrent operations $\operatorname{add}(m)$ and mult $(n)$, instead of requiring all replicas to apply add $(m)$ before mult $(n)$, we can equivalently require replicas that first received mult $(n)$ to apply add $(n m)$ when they later receive $\operatorname{add}(m)$. Replicas that instead received $\operatorname{add}(m)$ before mult $(n)$ apply both operations normally. This rule is inspired by the semidirect product of groups (see Appendix A), in which non-commuting operations from two groups can be transposed so long as we transform the first group's operations by the second's. 
Using this rule in the above scenario, both replicas end up in state 17:

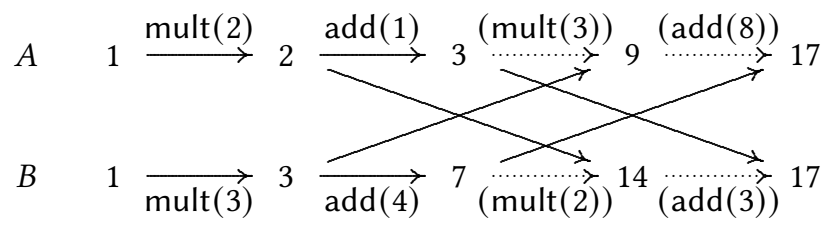

Here $A$ applies add(8) in place of add(4) because of its concurrent mult(2) operation, and $B$ applies add(3) in place of add(1) because of its concurrent mult(3) operation.

Unlike our first attempt, this approach generalizes to all situations via the rule: upon receiving an operation add $(m)$, instead of applying it directly, a replica applies the operation add $\left(n_{1} n_{2} \cdots n_{k} m\right)$, where mult $\left(n_{1}\right)$, mult $\left(n_{2}\right), \ldots$, mult $\left(n_{k}\right)$ are all of the mult operations concurrent to add $(m)$ that the replica had previously applied.

\subsection{Construction}

We obtain our semidirect product construction by generalizing the above approach. Let $C_{1}=$ $\left(\Sigma, \sigma^{0}\right.$, prepare $_{1}$, effect ${ }_{1}$, eval $)$ and $C_{2}=\left(\Sigma, \sigma^{0}\right.$, prepare $_{2}$, effect ${ }_{2}$, eval $)$ be two op-based CRDTs sharing the same state space, initial state, and eval function, but with disjoint sets of operations and prepared messages.

In addition to properties (i) and (ii) of an op-based CRDT, we assume that $C_{1}$ and $C_{2}$ satisfy the following property (iib), which is a strengthening of property (ii). Here by an author of a message $m$, we mean any replica $r$ such that $m=\operatorname{prepare}(o, \sigma, r)$ for some $o$ and $\sigma$, and we say two messages $m_{1}, m_{2}$ can have different authors if we can write $m_{1}=$ prepare $\left(o_{1}, \sigma_{1}, r_{1}\right)$ and $m_{2}=$ prepare $\left(o_{2}, \sigma_{2}, r_{2}\right)$ with $r_{1} \neq r_{2}$.

(iib) For all $\sigma \in \Sigma$ and all messages $m_{1}, m_{2}$ that can have different authors, if $m_{1} \cdot \sigma \neq \perp$ and $m_{2} \cdot \sigma \neq \perp$, then

$$
m_{1} \cdot\left(m_{2} \cdot \sigma\right)=m_{2} \cdot\left(m_{1} \cdot \sigma\right) \neq \perp .
$$

This is a stronger requirement than property (ii), which only requires $m_{1}$ and $m_{2}$ to commute if they can be sent concurrently in an execution of their parent CRDT alone. Instead, we require them to commute whenever they both make sense in state $\sigma$ and can have different authors. This strengthened version is necessary because in the semidirect product construction, we combine operations from $C_{1}$ and $C_{2}$. Thus it is possible that, for example, a $C_{2}$ message could lead to a state that is not reachable via $C_{1}$ messages alone, allowing a replica to issue a $C_{1}$ message $m_{1}$ that would never appear in an execution of $C_{1}$, which must then commute with $C_{1}$ messages $m_{2}$ sent concurrently in the semidirect product CRDT, even though property (ii) of $C_{1}$ does not require this Meanwhile, we only require $m_{1}$ and $m_{2}$ to commute if they can have different authors because a replica may have multiple choices of which operation to issue in a state $\sigma$, and the corresponding messages will both make sense in state $\sigma$, but there is no reason for them to commute. Indeed, after applying one message, the other may no longer make sense, for instance if they include identical timestamps.

Let $M_{1}$ and $M_{2}$ be the sets of prepared messages for $C_{1}$ and $C_{2}$, respectively. We wish to construct a CRDT combining the operations of $C_{1}$ and $C_{2}$, with $M_{1}$ coming before $M_{2}$ in the arbitration order, so that in the face of concurrent messages from the two CRDTs, those from $C_{1}$ are effectively applied first.

To make this possible, we assume we are given a partial action of $M_{2}$ on $M_{1}$, i.e., a partial function $\triangleright: M_{2} \times M_{1} \rightarrow M_{1}$. We write $\triangleright$ as in infix, e.g., $m_{2} \triangleright m_{1}$. This action will be used to transform a message $m_{1} \in M_{1}$ that is received after a concurrent message $m_{2} \in M_{2}$, to give the same result as if $m_{1}$ had been applied before $m_{2}$ like it should have been. 
Example 3.1. In the example of the previous section, $C_{1}$ is an integer register with operations $\operatorname{add}(m), C_{2}$ is an integer register with operations mult $(n)$, and $\triangleright$ is given by

$$
\operatorname{mult}(n) \triangleright \operatorname{add}(m)=\operatorname{add}(n m) .
$$

For the semidirect product to work, we assume:

(reordering) For all $\sigma \in \sum$ and all messages $m_{1} \in M_{1}, m_{2} \in M_{2}$ that can have different authors (i.e., we can write $m_{1}=$ prepare $_{1}\left(o_{1}, \sigma_{1}, r_{1}\right)$ and $m_{2}=$ prepare $_{2}\left(o_{2}, \sigma_{2}, r_{2}\right)$ with $\left.r_{1} \neq r_{2}\right)$, if $m_{1} \cdot \sigma \neq \perp$ and $m_{2} \cdot \sigma \neq \perp$, then $m_{2} \triangleright m_{1} \neq \perp$ and

$$
m_{2} \cdot\left(m_{1} \cdot \sigma\right)=\left(m_{2} \triangleright m_{1}\right) \cdot\left(m_{2} \cdot \sigma\right) \neq \perp .
$$

This ensures that in case of concurrent messages $m_{1}$ and $m_{2}$ acting on a state $\sigma$, the intended final state $m_{2} \cdot\left(m_{1} \cdot \sigma\right)$ is defined, and it can be computed either by applying $m_{1}$ followed by $m_{2}$ or by applying $m_{2}$ followed by $m_{2} \triangleright m_{1}$.

(action commutes) For all $m_{1} \in M_{1}$ and all $m_{2}, m_{2}^{\prime} \in M_{2}$, if $m_{1}, m_{2}, m_{2}^{\prime}$ can have mutually different authors, then

$$
m_{2} \triangleright\left(m_{2}^{\prime} \triangleright m_{1}\right)=m_{2}^{\prime} \triangleright\left(m_{2} \triangleright m_{1}\right) .
$$

This ensures that concurrent $M_{2}$ messages commute in their action on $M_{1}$ messages via $\triangleright$, just like how they commute when applied to states in $\Sigma$.

(preserves authors) For all $m_{1}=$ prepare $_{1}\left(o_{1}, \sigma_{1}, r_{1}\right)$ and $m_{2} \in M_{2}$, if $m_{2} \triangleright m_{1} \neq \perp$, then $m_{2} \triangleright m_{1}=$ prepare $_{1}\left(o_{1}^{\prime}, \sigma_{1}^{\prime}, r_{1}\right)$ for some $o_{1}^{\prime}, \sigma_{1}^{\prime}$. This ensures that $\triangleright$ preserves message authors, hence messages $m_{1}^{\prime} \in M_{1}$ that are required to commute with $m_{1}$ by property (iib) of an op-based CRDT are also required to commute with $m_{2} \triangleright m_{1}{ }^{1}$

Example 3.2. In the example of the previous section, assumption (reordering) holds by the distributive property:

$$
\begin{array}{r}
\operatorname{mult}(n) \cdot(\operatorname{add}(m) \cdot \sigma)=n(m+\sigma)=n m+n \sigma \\
=\operatorname{add}(m n) \cdot(\operatorname{mult}(n) \cdot \sigma) .
\end{array}
$$

Assumption (action commutes) holds by commutativity of multiplication, and (preserves authors) is trivial as any message can have any author.

Definition 3.3. Given the assumptions above, the semidirect product of $C_{1}$ and $C_{2}$ with respect to $\triangleright$ is the op-based CRDT $C_{1} \rtimes_{\triangleright} C_{2}=\left(\Sigma_{\rtimes},\left(\sigma^{0}, \mathbf{0}, \emptyset\right)\right.$, prepare ${ }_{\rtimes}$, effect $_{\rtimes}$, eval $\left.\left.\right|_{\rtimes}\right)$ with components defined in Algorithm 2.

The semidirect product functions as follows. A state $(\sigma, t, H)$ corresponds to an internal state $\sigma$ with current timestamp $t$ and history of $M_{2}$ messages $H$. To apply a message $m \in M_{2}$, effect ${ }_{\rtimes}$ applies $m$ to $\sigma$ and also stores $m$ in $H$ together with its timestamp. To apply a message $m \in M_{1}$, effect $_{\rtimes}$ first acts on $m$ by all concurrent messages in $H$, i.e., all concurrent $M_{2}$ messages that have already been applied to the state, using timestamps to determine which messages are concurrent. These concurrent messages $\left(l_{1}, u_{1}\right), \ldots,\left(l_{k}, u_{k}\right)$ act on $m$ in causal order, by which we mean any ordering such that for all $j<j^{\prime}, u_{j^{\prime}}$ is not causally prior to $u_{j}$. The resulting message $m_{\text {act }}$ is then applied to $\sigma$.

\footnotetext{
${ }^{1}$ In practice, $m_{2} \triangleright m_{1}$ sometimes exists only for the purposes of the semidirect product and is not an output of prepare ${ }_{1}$, in which case we formally define its author to be that of $m_{1}$, for the purposes of property (iib) and the above assumptions.
} 


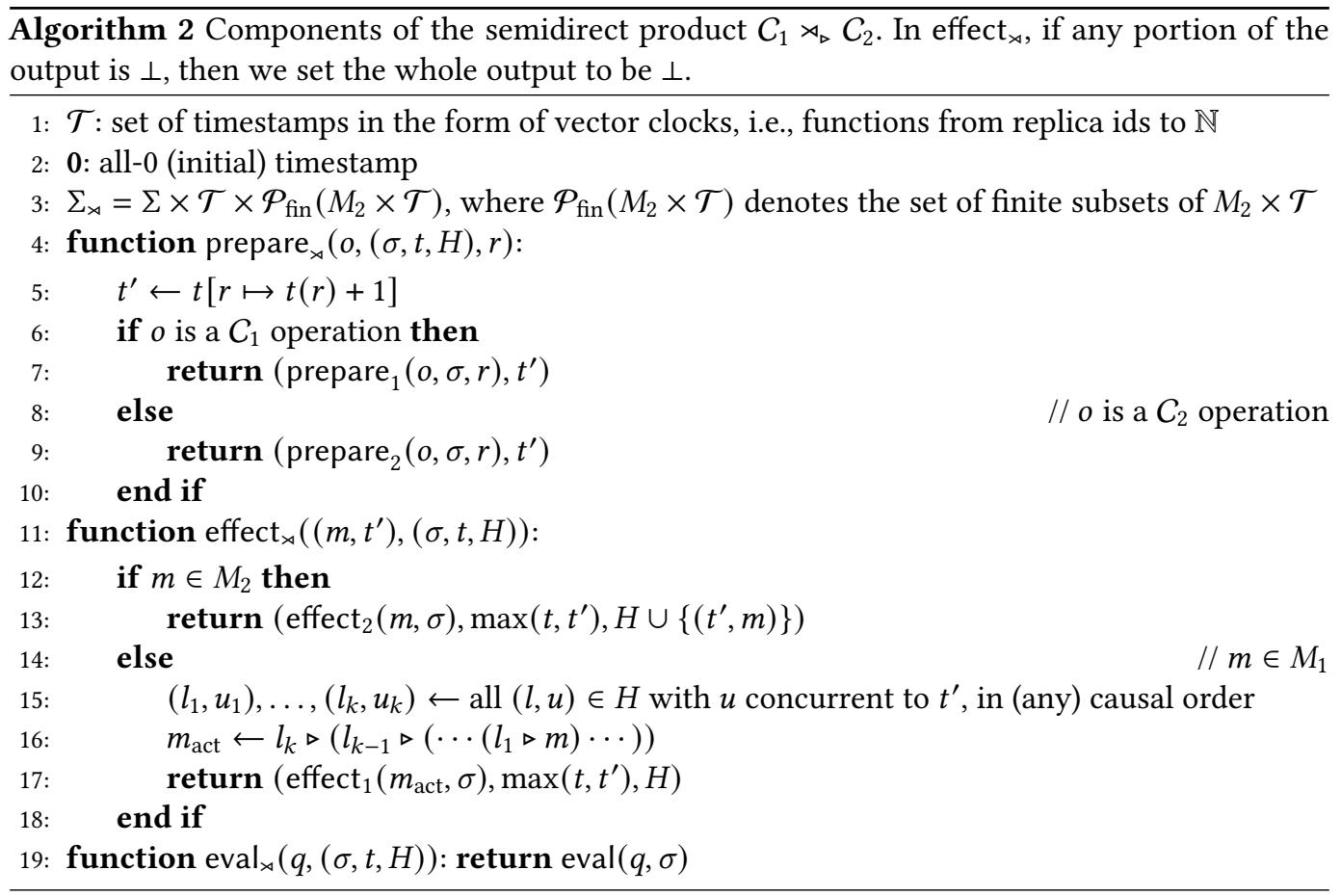

\subsection{Correctness}

Theorem 3.4. $C_{1} \rtimes_{\triangleright} C_{2}$ is an op-based CRDT in the sense of Definition 2.2.

Proof. First, note that $\left(m, t^{\prime}\right) \cdot(\sigma, t, H)$ is the same regardless of which causal ordering we choose for $\left(l_{1}, u_{1}\right), \ldots,\left(l_{k}, u_{k}\right)$ on line 15 . Indeed, if $\left(l_{1}^{\prime}, u_{1}^{\prime}\right), \ldots,\left(l_{k}^{\prime}, u_{k}^{\prime}\right)$ is another causal ordering of these messages, then we can go from one sequence to the other via a sequence of transpositions

$$
\left(l_{j_{1}}, u_{j_{1}}\right),\left(l_{j_{2}}, u_{j_{2}}\right) \mapsto\left(l_{j_{2}}, u_{j_{2}}\right),\left(l_{j_{1}}, u_{j_{1}}\right)
$$

with $u_{j_{1}}$ concurrent to $u_{j_{2}}$. Since $u_{j_{1}}, u_{j_{2}}$, and $t$ are mutually concurrent, $l_{j_{1}}, l_{j_{2}}$, and $m$ must have had mutually different authors in the execution leading to this state. Hence by assumption (action commutes), such a transposition does not change $m_{\text {act }}$.

We now verify the CRDT properties. Recall that we use $m \cdot \sigma$ as an abbreviation for $\operatorname{effect}(m, \sigma)$ when the relevant CRDT is clear from context.

(i) Let $\left(m, t^{\prime}\right)=\operatorname{prepare}_{\rtimes}(o,(\sigma, t, H), r)$. If $m \in M_{2}$, then $m \cdot \sigma \neq \perp$ by property (i) of $C_{2}$, so $\left(m, t^{\prime}\right) \cdot(\sigma, t, H) \neq \perp$. If instead $m \in M_{1}$, then there are no $(l, u) \in H$ with $u$ concurrent to $t^{\prime}$, since $t^{\prime}$ comes causally after all prior timestamps. Hence $m_{\text {act }}=m$, so $m_{\text {act }} \cdot \sigma=m \cdot \sigma \neq \perp$ by property (i) of $C_{1}$. Thus $\left(m, t^{\prime}\right) \cdot(\sigma, t, H) \neq \perp$.

(ii) Let $(\sigma, t, H) \in \Sigma_{\rtimes}$ be a state and $\left(l, s^{\prime}\right),\left(m, t^{\prime}\right)$ be messages such that $(\sigma, t, H)$ can appear as a replica state and $\left(l, s^{\prime}\right)$ and $\left(m, t^{\prime}\right)$ can appear as concurrent messages in an execution of Algorithm 1 , and $\left(l, s^{\prime}\right) \cdot(\sigma, t, H) \neq \perp$ and $\left(m, t^{\prime}\right) \cdot(\sigma, t, H) \neq \perp$. We need to show that

$$
\left(l, s^{\prime}\right) \cdot\left(\left(m, t^{\prime}\right) \cdot(\sigma, t, H)\right)=\left(m, t^{\prime}\right) \cdot\left(\left(l, s^{\prime}\right) \cdot(\sigma, t, H)\right) \neq \perp .
$$

Case $l, m \in M_{1}$ : Let $l_{\text {act }}$ and $m_{\text {act }}$ be as in the definitions of $\left(l, s^{\prime}\right) \cdot(\sigma, t, H)$ and $\left(m, t^{\prime}\right) \cdot(\sigma, t, H)$, respectively. By assumption (preserves authors) and property (iib) of $C_{1}, l_{\text {act }}$ and $m_{\text {act }}$ commute when applied to $\sigma$, and the claim follows. 
Case $l, m \in M_{2}$ : By property (iib) of $C_{2}, l$ and $m$ commute when applied to $\sigma$, and the claim follows.

Case $l \in M_{1}, m \in M_{2}$ : Let $l_{\text {act }}$ and $l_{\text {act }}^{\prime}$ be as in the definitions of $\left(l, s^{\prime}\right) \cdot(\sigma, t, H)$ and $\left(l, s^{\prime}\right) \cdot\left(\left(m, t^{\prime}\right) \cdot(\sigma, t, H)\right)$, respectively. Since $s^{\prime}$ is concurrent to $t^{\prime},\left(m, t^{\prime}\right)$ appears among the operations used to compute $l_{\text {act }}^{\prime}$. Furthermore, since Algorithm 1 delivers messages in causal order, $t^{\prime}$ must be maximal among timestamps in $H$, so we can put $m$ last in the sequence used to define $l_{\text {act }}^{\prime}$. Thus $l_{\text {act }}^{\prime}=m \triangleright l_{\text {act }}$. Also, $l_{\text {act }} \cdot \sigma \neq \perp$ and $m \cdot \sigma \neq \perp$, and by assumption (preserves authors), $l_{\text {act }}$ and $m$ can have different authors. Hence by assumption (reordering), $m \cdot\left(l_{\text {act }} \cdot \sigma\right)=\left(m \triangleright l_{\text {act }}\right) \cdot(m \cdot \sigma) \neq \perp$. The claim follows.

\section{GUIDE TO USING SEMIDIRECT PRODUCTS}

In this section, we go through the steps a CRDT designer should use to construct a semidirect product CRDT. We do so in the context of an example promised in the introduction: adding map operations to a dictionary CRDT. Additional examples appear in Section 5.

Let $C$ be a CRDT and $K$ be a set of keys. Numerous works [AntidoteDB 2019; Basho 2015; Kleppmann and Beresford 2016] define a dictionary CRDT $C_{\text {dict }}$ with keys $K$ and values in $C$, which is a replicated version of a dictionary. The states of $C_{\text {dict }}$ are partial functions $f$ from $K$ to states of $C$ such that all but finitely many values of $f$ are $\perp$. The operations are of the form apply $(k, m)$ for $k \in K$ and $m$ a message of $C$, with effect

$$
(\operatorname{apply}(k, m) \cdot f)\left(k^{\prime}\right)= \begin{cases}f\left(k^{\prime}\right) & \text { if } k^{\prime} \neq k \\ m \cdot f(k) & \text { if } k^{\prime}=k \text { and } f(k) \neq \perp \\ m \cdot \sigma^{0} & \text { if } k^{\prime}=k \text { and } f(k)=\perp .\end{cases}
$$

That is, apply $(k, m)$ applies $m$ to the value at $k$, treating $\perp$ as $C$ 's initial state $\sigma^{0}$. Typically, CRDT dictionaries also include an operation to remove a key-value pair, but incorporating such an operation into our construction below is difficult, so we leave it as future work.

For our novel CRDT, we wish to add map operations $\operatorname{map}\left(m^{\prime}\right)$ to $C_{\text {dict }}$, where $m^{\prime}$ is a message of $C$, with sequential semantics

$$
\left(\operatorname{map}\left(m^{\prime}\right) \cdot f\right)(k)= \begin{cases}\perp & \text { if } f(k)=\perp \\ m^{\prime} \cdot f(k) & \text { otherwise. }\end{cases}
$$

That is, $\operatorname{map}\left(m^{\prime}\right)$ applies $m^{\prime}$ to every non- $\perp$ value in the dictionary. ${ }^{2}$

Example 4.1. As a potential use case, recall the example from the introduction of a Slack-like application containing multiple "channels" that users can join. We can store the mapping from channel names to the set of users in each channel as a set-valued dictionary CRDT. When a new user $A$ is added to the application, we can use map $(\operatorname{add}(A))$ to add $A$ to every channel, without needing to send separate messages for each channel. It is trivial to modify the operation to filter by keys, so that, e.g., $A$ is only added to channels whose names start with "public".

As our first step in constructing a semidirect product implementing both apply and map operations, we need to partition the operations into two sets so that we can easily construct CRDTs for each set. Here the obvious split is into apply and map operations. Thus our first component CRDT is $C_{\text {dict }}$, which implements the apply operations. For our second component CRDT, we let $C_{\text {map }}$ have the same state space as $C_{\text {dict }}$ but with map operations only. Since $C$ is a CRDT, concurrent map $\left(m^{\prime}\right)$

\footnotetext{
${ }^{2}$ Strictly speaking, the allowable choices of $m^{\prime}$ should be restricted so that $m^{\prime} \cdot f(k) \neq \perp$ for all $k$, and so that $m^{\prime}$ commutes with messages $m$ that could appear in a concurrent $\operatorname{map}(m)$ or apply $(k, m)$ operation.
} 
operations commute with each other, so $C_{\text {map }}$ is indeed a CRDT. Also, assuming $C$ satisfies the strengthened property (iib) of an op-based CRDT, so do $C_{\text {dict }}$ and $C_{\text {map }}$.

Note that we cannot add $\operatorname{map}\left(m^{\prime}\right)$ operations to $C_{\text {dict }}$ directly, since they do not always commute with concurrent operations apply $(k, m)$. Indeed, suppose apply $(k, m)$ initializes the value at $k$, i.e., changes it from $\perp$ to non- $\perp$. This does not commute with a concurrent operation $\operatorname{map}\left(m^{\prime}\right)$ : $\operatorname{map}\left(m^{\prime}\right)$ will only modify the value at $k$ if it is applied after apply $(k, m)$, not before, since it only affects initialized values.

Hence we are led to consider a semidirect product of $C_{\text {dict }}$ and $C_{\text {map }}$. To do so, we must first choose an arbitration order between the two CRDTs. That is, we must choose which operation should be applied first in the case of concurrent apply $(k, m)$ and $\operatorname{map}\left(m^{\prime}\right)$ operations. Either choice would be reasonable, but we find it more interesting to put $\operatorname{map}\left(\mathrm{m}^{\prime}\right)$ operations last in the arbitration order. This means that they also apply to keys that are initialized concurrently.

Example 4.1 cont. In the Slack-like application example, when using map $(\operatorname{add}(A))$ to add $A$ to every channel, this semantics ensures that $A$ will also be added to channels that are created concurrently to $A$ 's addition, so that they are not left out of any eligible channels.

Next, we need to choose a partial action $\triangleright$ of $\operatorname{map}\left(m^{\prime}\right)$ messages on apply $(k, m)$ messages satisfying assumption (reordering), i.e., for all dictionary states $f$,

$$
\operatorname{map}\left(m^{\prime}\right) \cdot(\operatorname{apply}(k, m) \cdot f)=\left(\operatorname{map}\left(m^{\prime}\right) \triangleright \operatorname{apply}(k, m)\right) \cdot\left(\operatorname{map}\left(m^{\prime}\right) \cdot f\right) .
$$

We focus on this assumption first because it is the most difficult one to satisfy, while assumptions (action commutes) and (preserves authors) are largely technical. In general, to satisfy this assumption, we often need to modify our component CRDTs $C_{1}$ and $C_{2}$ :

- Sometimes for messages $m_{1}$ of $C_{1}$ and $m_{2}$ of $C_{2}$, there is an obvious partial function $l$ from states to states such that $m_{2} \cdot\left(m_{1} \cdot \sigma\right)=l \cdot\left(m_{2} \cdot \sigma\right)$ for all states $\sigma$, but $l$ does not correspond to the effect of any message of $C_{1}$. This is easily resolved by expanding the message set of $C_{1}$ to include $l$. These "ghost" messages need not have a corresponding externally visible operation. Note, however, that we must then ensure that assumption (reordering) and the CRDT property (iib) also hold for these new messages of $C_{1}$.

- Sometimes it is impossible to find a function $l$ as above, or all such $l$ give the wrong semantics. This can often be solved by adding extra metadata to states that gives us more flexibility in our choice of $l$.

We end up needing both of these techniques here, making it a rather complicated example; our other examples of novel CRDTs in Section 5.1 are simpler, requiring only trivial modifications to the component CRDTs.

To find $\triangleright$ satisfying (1), observe that apply $(k, m)$ and $\operatorname{map}\left(m^{\prime}\right)$ already commute when applied to a state with $f(k) \neq \perp$. Indeed, in either order, they set the value at $k$ to $m \cdot\left(m^{\prime} \cdot f(k)\right)=m^{\prime} \cdot(m \cdot f(k))$. Thus when $f(k) \neq \perp$, we want $\operatorname{map}\left(m^{\prime}\right) \triangleright$ apply $(k, m)$ to have the same effect as apply $(k, m)$. However, when $f(k)=\perp$, we want $\operatorname{map}\left(m^{\prime}\right) \triangleright \operatorname{apply}(k, m)$ to apply both $m$ and $m^{\prime}$ to the newly initialized value at $k$, since this is what happens in $\operatorname{map}\left(m^{\prime}\right) \cdot(\operatorname{apply}(k, m) \cdot f)$.

No apply message acts in this way, but as discussed in the first bullet above, we can easily add a message that does. Specifically, we can define a new message apply $(k, m)\left(m^{\prime}\right)$ whose effect is to apply both $m$ and $m^{\prime}$ to the value at $k$ if it is uninitialized, otherwise just applying $m$. Then setting

$$
\operatorname{map}\left(m^{\prime}\right) \triangleright \operatorname{apply}(k, m)=\operatorname{apply}(k, m)\left(m^{\prime}\right)
$$

satisfies (1).

However, these new messages are also messages of $C_{\text {dict }}$, so we need to define $\triangleright$ on them as well. The action should have the same form as before: $\operatorname{map}\left(m_{2}^{\prime}\right)$ should act on apply $(k, m)\left(m_{1}^{\prime}\right)$ to 
give a message that applies all of $m, m_{1}^{\prime}$, and $m_{2}^{\prime}$ to the value at $k$ if it is uninitialized, otherwise just applying $m$. Continuing in this fashion, we see that in general, we want to add messages apply $(k, m)\left(L^{\prime}\right)$ to $C_{\text {dict }}$, where $L^{\prime}$ is a finite sequence of $C$ messages. The effect of apply $(k, m)\left(L^{\prime}\right)$ is to apply $m$ and all messages in $L^{\prime}$ to the value at $k$ if it is uninitialized, otherwise just applying $m$. Then we can define $\triangleright$ by

$$
\operatorname{map}\left(m^{\prime}\right) \triangleright \operatorname{apply}(k, m)\left(L^{\prime}\right)=\operatorname{apply}(k, m)\left(L^{\prime} \cup\left\{m^{\prime}\right\}\right) .
$$

These new messages have the problem that they do not commute with each other. Indeed, if $m_{1}^{\prime} \neq m_{2}^{\prime}$, then applying apply $\left(m_{1}\right)\left(\left[m_{1}^{\prime}\right]\right)$ followed by a concurrent message apply $\left(m_{2}\right)\left(\left[m_{2}^{\prime}\right]\right)$ to a state with $f(k)=\perp$ gives a state with value $m_{2} \cdot\left(m_{1} \cdot\left(m_{1}^{\prime} \cdot \sigma^{0}\right)\right)$ at $k$, while the opposite order gives $m_{2} \cdot\left(m_{1} \cdot\left(m_{2}^{\prime} \cdot \sigma^{0}\right)\right)$. To solve this, we want to apply both $m_{1}^{\prime}$ and $m_{2}^{\prime}$ to the value at $k$, but only if they have not already been applied by the messages map $\left(m_{1}^{\prime}\right)$ or map $\left(m_{2}^{\prime}\right)$. This is impossible with our current state space, but as discussed in the second bullet above, it is easily solved by adding extra metadata to states. Specifically, we can have our states track which map $\left(m^{\prime}\right)$ messages have already been applied to the value at $k$, for each $k \in K$. Then apply $(k, m)\left(L^{\prime}\right)$ should apply any messages from $L^{\prime}$ that have not already been applied, regardless of whether the value at $k$ is already initialized. Thus a $\operatorname{map}\left(m^{\prime}\right)$ operation issued concurrently to any apply $(k, m)$ operation-not just the first one received by a replica-will eventually be applied to the value at $k$.

Formally, we define the states of a modified CRDT $C_{\text {dict }}^{\prime}$ to be partial functions $g$ from $K$ to pairs $(\sigma, L)$ such that all but finitely many values of $g$ are $\perp$. We require that in each value $g(k)=(\sigma, L)$, $L$ is a finite causally ordered sequence of $C$ messages $^{3}$ and $\sigma=L \cdot \sigma^{0}$, where $L \cdot \sigma^{0}$ denotes the result of applying all messages in $L$ to $\sigma^{0}$ in causal order. The externally visible dictionary value $f$ corresponding to $g$ is given by the $\sigma$ coordinates. The messages of $C_{\text {dict }}^{\prime}$ are of the form apply $(k, m)\left(L^{\prime}\right)$ for $k \in K, m$ a message of $C$, and $L^{\prime}$ a finite sequence of $C$ messages. The effect of such a message on a state $g$ is

$$
\left(\operatorname{apply}(k, m)\left(L^{\prime}\right) \cdot g\right)\left(k^{\prime}\right)= \begin{cases}g\left(k^{\prime}\right) & \text { if } k^{\prime} \neq k \\ (m \cdot \sigma, L \cup\{m\}) & \text { if } k^{\prime}=k, g(k)=(\sigma, L), \text { and } L^{\prime} \subset L \\ \left(m \cdot\left(\left(L \cup L^{\prime}\right) \cdot \sigma^{0}\right),\right. & \\ \left.L \cup L^{\prime} \cup\{m\}\right) & \text { if } k^{\prime}=k, g(k)=(\sigma, L), \text { and } L^{\prime} \not \subset L \\ \left(m \cdot\left(L \cdot \sigma^{0}\right), L \cup\{m\}\right) & \text { if } k^{\prime}=k \text { and } g(k)=\perp .\end{cases}
$$

In other words, apply $(k, m)(L)$ acts the same as apply $(k, m)$, except it also applies any messages in $L$ that have not already been applied. The original dictionary operations apply $(k, m)$ correspond to the messages apply $(k, m)(\emptyset)$.

We define a modified CRDT $C_{\text {map }}^{\prime}$, which has the same state space as $C_{\text {dict }}^{\prime}$ but map operations only, by setting

$$
\left(\operatorname{map}\left(m^{\prime}\right) \cdot g\right)(k)= \begin{cases}\perp & \text { if } g(k)=\perp \\ \left(m^{\prime} \cdot \sigma, L \cup\left\{m^{\prime}\right\}\right) & \text { if } g(k)=(\sigma, L) .\end{cases}
$$

One can check that both $C_{\text {dict }}^{\prime}$ and $C_{\text {map }}^{\prime}$ are op-based CRDTs with the strengthened property (iib), and they preserve the invariant that $\sigma=L \cdot \sigma^{0}$ for each value $g(k)=(\sigma, L)$.

Now we can define

$$
\operatorname{map}\left(m^{\prime}\right) \triangleright \operatorname{apply}(k, m)\left(L^{\prime}\right)=\operatorname{apply}(k, m)\left(L^{\prime} \cup\left\{m^{\prime}\right\}\right) .
$$

\footnotetext{
${ }^{3}$ Technically, we should use unique identifiers to distinguish between messages coming from distinct operations apply $(k, m)$ or map $(m)$ with the same argument $m$.
} 
This trivially satisfies assumptions (action commutes) and (preserves authors), and it satisfies assumption (reordering) as well: both

$$
\left(\operatorname{map}\left(m^{\prime}\right) \cdot\left(\operatorname{apply}(k, m)\left(L^{\prime}\right) \cdot g\right)\right)(k)
$$

and

$$
\left(\operatorname{apply}(k, m)\left(L^{\prime} \cup\{m\}\right) \cdot\left(\operatorname{map}\left(m^{\prime}\right) \cdot g\right)\right)(k)
$$

equal

$$
\begin{cases}\left(\left(\left\{m, m^{\prime}\right\} \cup L^{\prime} \cup L\right) \cdot \sigma^{0},\left\{m, m^{\prime}\right\} \cup L^{\prime} \cup L\right) & \text { if } g(k)=(\sigma, L) \\ \left(\left(\left\{m, m^{\prime}\right\} \cup L^{\prime}\right) \cdot \sigma^{0},\left\{m, m^{\prime}\right\} \cup L^{\prime}\right) & \text { if } g(k)=\perp .\end{cases}
$$

Thus we get a semidirect product CRDT $C_{\text {dict }}^{\prime} \rtimes_{\triangleright} C_{\text {map }}^{\prime}$ with both apply $(k, m)$ and map $\left(m^{\prime}\right)$ operations, implementing the semantics described above. In this CRDT, a map $\left(m^{\prime}\right)$ operation modifies concurrent apply $\left(k, m^{\prime}\right)$ operations to ensure that $m^{\prime}$ will be applied to the value at $k$ if it has not been applied already.

\section{EXAMPLES}

We now give numerous examples of semidirect products, both constructing novel CRDTs and reproducing the semantics of existing CRDTs. These examples demonstrate the semidirect product's ability to compose and decompose CRDTs. In several of the examples, both components are commutative data types (i.e., all messages commute naturally without conflict resolution), in which case the semidirect product handles all conflicts between non-commuting concurrent messages.

\subsection{Novel CRDTs}

Sequence with Reverse Operation. We can use semidirect products to add a reverse operation to a sequence CRDT. A sequence CRDT is a CRDT version of a totally ordered sequence, such as a text string, as would appear in a collaborative text editing application.

Let $C$ be the continuous sequence CRDT defined by Shapiro et al. [2011, §3.5.2], with $\mathbb{R}$ as the continuum set of identifiers. In this CRDT, the state consists of a set $S$ of sequence elements (e.g., characters) tagged with unique identifiers of the form $(x, r)$ for $x \in \mathbb{R}$ and $r$ a replica id. The identifiers, and their corresponding elements, are totally ordered by their real number component, with ties broken using an arbitrary total order on replica ids. Calling prepare on an operation to insert an element $e$ to the right of a given element $e^{\prime}$ results in a message add $(e,(x, r))$, where $r$ is the generating replica's id and $x$ is a real number halfway between the identifiers for $e^{\prime}$ and the next element to the right of $e^{\prime}$. The effect of $\operatorname{add}(e,(x, r))$ is to add $e$ to the state with identifier $(x, r)$. We also have operations remove $(x, r)$, which remove the element with identifier $(x, r)$.

For our novel CRDT, we wish to add an operation reverse acting as

$$
\text { reverse } \cdot S=\{(e,(-x,-r)) \mid(e,(x, r)) \in S\},
$$

where "negative" replica ids are ordered oppositely to ordinary replica ids. Let $C_{\text {rev }}$ be the commutative data type with the same state space as $C$ and with reverse as its single operation and message. We choose reverse to come after messages of $C$ in the arbitration order, so that concurrent insertions are also reversed. Next, we must find an action $\triangleright$ of reverse on messages of $C$ satisfying assumption (reordering). This is easily done by setting

$$
\begin{aligned}
& \text { reverse } \triangleright \operatorname{add}(e,(x, r))=\operatorname{add}(e,(-x,-r)) \\
& \text { reverse } \triangleright \operatorname{remove}(x, r)=\operatorname{remove}(-x,-r) .
\end{aligned}
$$

Assumptions (action commutes) and (preserves authors) hold as well. Thus the semidirect product CRDT $C \rtimes_{\triangleright} C_{\text {rev }}$ implements the operations of $C$ together with reverse. 
Remark 5.1. It is also possible to use the opposite arbitration order, in which reverse operations come first and hence do not affect concurrent operations, using a semidirect product in which a message $m$ of $C$ acts on a reverse operation to add an exception for $m$. We omit the details.

Sequence with Range Remove Operation. Let $C$ be the continuous sequence CRDT described above. We can also use the semidirect product to add a range remove operation rremove $\left((x, r),\left(x^{\prime}, r^{\prime}\right)\right)$ to $C$, which removes all elements with identifiers $(x, r) \leq(y, s) \leq\left(x^{\prime}, r^{\prime}\right)$. This could be useful as an optimization in a collaborative text editor when a user highlights and deletes a block of text, in place of sending separate remove messages for each character.

Let $C_{\text {rremove }}$ be the commutative data type with the same state space as $C$ and with operations rremove $\left((x, r),\left(x^{\prime}, r^{\prime}\right)\right)$, acting as described above. We choose rremove operations to come after messages of $C$ in the arbitration order, so that they affect concurrent additions. We define $\triangleright$ by

$$
\begin{aligned}
& \operatorname{rremove}\left((x, r),\left(x^{\prime}, r^{\prime}\right)\right) \triangleright \operatorname{add}(e,(y, s)) \\
& = \begin{cases}\operatorname{id} & \text { if }(x, r) \leq(y, s) \leq\left(x^{\prime}, s^{\prime}\right) \\
\operatorname{add}(e,(y, s)) & \text { otherwise }\end{cases} \\
& \operatorname{rremove}\left((x, r),\left(x^{\prime}, r^{\prime}\right)\right) \triangleright \operatorname{remove}(y, s)=\operatorname{remove}(y, s),
\end{aligned}
$$

where id is a new message we add to $C$ which acts as the identity. Then the semidirect product assumptions are easily checked, so we get a semidirect product CRDT $C \rtimes_{\triangleright} C_{\text {rremove }}$ implementing the operations of $C$ together with rremove.

As with reverse, it is also possible to implement the opposite arbitration order, in which rremove operations only remove elements that were added causally before the rremove.

Semirings. The example of Section 3.1 generalizes to the case when we replace $(\mathbb{Z},+, \times)$ by any commutative semiring:

Definition 5.2. A commutative semiring [Rosenfeld 1968] is a tuple $(S, \oplus, \otimes)$ consisting of a set of states $S$ and binary operations $\oplus, \otimes: S \times S \rightarrow S$, such that $\oplus$ and $\otimes$ are associative and commutative, and $\otimes$ distributes over $\oplus$, i.e., for all $s, t, u \in S, s \otimes(t \oplus u)=(s \otimes t) \oplus(s \otimes u)$.

Examples include $(\mathbb{Z},+, \times),(\mathbb{N}, \min ,+),(\mathbb{N}, \max , \min )$, and $(\mathbb{N}, \min , \max )$. We will define a semidirect product CRDT implementing the operations of any commutative semiring, from which we immediately get CRDTs implementing all of these examples.

Given a commutative semiring $(S, \oplus, \otimes)$, let $C_{1}$ be the commutative data type with state space $S$ and operations add $(s), s \in S$, acting as add $(s) \cdot \sigma=s \oplus \sigma$. Similarly let $C_{2}$ be the commutative data type with state space $S$ and operations mult $(t), t \in S$, acting as mult $(t) \cdot \sigma=t \otimes \sigma$. Following the example of Section 3.1, we can define $\triangleright$ by

$$
\operatorname{mult}(t) \triangleright \operatorname{add}(s)=\operatorname{add}(t \otimes s),
$$

and then we get a semidirect product CRDT $C_{1} \rtimes_{\triangleright} C_{2}$. Here assumption (reordering) holds by the distributive property:

$$
\begin{array}{r}
\operatorname{mult}(t) \cdot(\operatorname{add}(s) \cdot \sigma)=t \otimes(s \oplus \sigma)=(t \otimes s) \oplus(t \otimes \sigma) \\
=\operatorname{add}(t \otimes s) \cdot(\operatorname{mult}(t) \cdot \sigma) .
\end{array}
$$

The semidirect product CRDT implements both add $(s)$ and mult $(t)$ operations on $S$, with mult $(t)$ operations affecting the arguments to concurrent add(s) operations.

Remark 5.3. By iteratively applying a semidirect product construction similar to the semiring construction, it appears to be possible to construct a CRDT supporting operations mult, add, min, 
and max on a natural number register, with arbitration order mult $>$ add $>\min >\max$. We leave the details to future work.

\subsection{Existing CRDTs}

Boolean Flags. The enable-wins flag [Basho 2015] is a simple CRDT with state space $\Sigma=$ \{enabled, disabled\}, initial state disabled, and operations enable and disable, with sequential semantics

$$
\text { enable } \cdot \sigma=\text { enabled, } \quad \text { disable } \cdot \sigma=\text { disabled }
$$

for all $\sigma \in \sum$. In case of concurrent enable and disable operations, the enable wins, so that the state is enabled. More precisely, letting $<$ be the causal order on messages, a replica's state is enabled if it has received any messages $m=$ enable such that it has not received any messages $m^{\prime}=$ disable with $m<m^{\prime}$; otherwise the state is disabled.

As our first step in constructing a semidirect product with the same semantics as the enable-wins flag, we need to partition the operations into two sets so that we can easily construct CRDTs for each set. Since there are only two operations, we take the two sets to be $\{$ enable $\}$ and $\{$ disable . Both of these singleton sets of operations, acting on the original state space $\Sigma$, define commutative data types (i.e., all messages commute naturally without conflict resolution), since trivially enable commutes with itself and likewise for disable. Hence they also define op-based CRDTs satisfying the strengthened property (iib).

Next, we must choose an arbitration order between the two CRDTs. That is, we must choose which operation should be applied first in the case of concurrent enable and disable operations. The enable-wins semantics corresponds to disable going first, so that the subsequent enable wins. Thus we take $C_{1}$ to be the CRDT with operation set $\left\{\right.$ disable\}, while $C_{2}$ is the CRDT with operation set $\{$ enable $\}$.

Next, we need to find a partial action $\triangleright$ of $C_{2}$ messages on $C_{1}$ messages satisfying assumption (reordering). As a first attempt, we add an identity message id to the message set of $C_{1}$, acting as id $\cdot \sigma=\sigma$, and define

$$
\text { enable } \triangleright \text { disable }=\text { id, } \quad \text { enable } \triangleright \text { id }=\text { id } .
$$

This satisfies assumption (reordering) because

$$
\text { enable } \circ \text { disable }=\text { enable }=\text { id } \circ \text { enable. }
$$

It also trivially satisfies assumptions (action commutes) and (preserves authors), where we formally allow any replica to be an author of id. Hence the semidirect product $C_{1} \rtimes_{\triangleright} C_{2}$ is defined. However, it does not quite implement the enable-wins semantics in the following scenario (using $\mathrm{d}$ and e as obvious abbreviations):

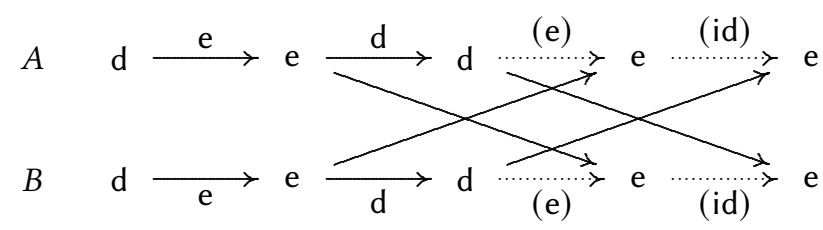

Here replicas $A$ and $B$ both start in state disabled, then each concurrently issue operations enable followed by disable. After receiving each others' messages, the intended result is disabled, since both enable operations have been overwritten by causally greater disable operations. However, the disable messages instead both get transformed to id by the concurrent enable messages, resulting in state enabled. 
To resolve this, observe that in the enable-wins flag, the effect of a disable message is not always to set the state to disabled. Instead, it cancels the effect of any causally lesser enable messages, resulting in state disabled only if no enable messages remain.

This motivates us to replace $\Sigma$ with the state space $\Sigma^{\prime}$ whose states are sets $S$ of enable messages. The externally visible value of a state $S$ is enabled if $S \neq \emptyset$ and disabled if $S=\emptyset$. In place of $C_{1}$, we define the CRDT $C_{1}^{\prime}$ which has a single operation and message disable acting as disable $\cdot S=\emptyset$. In place of $C_{2}$, we define the CRDT $C_{2}^{\prime}$ which has a single operation enable and message space $M_{2}^{\prime}$ containing infinitely many messages of the form enable ${ }^{4}$, acting as $m \cdot S=S \cup\{m\}$. Note that both $C_{1}^{\prime}$ and $C_{2}^{\prime}$ are commutative data types.

We once again choose $C_{1}^{\prime}$ (the disable operations) to come first in the arbitration order. Thus the semidirect product transformation $\triangleright^{\prime}$ must satisfy, for $m \in M_{2}^{\prime}$ and $s \in \Sigma^{\prime}$,

$$
m \cdot(\text { disable } \cdot S)=\left(m \triangleright^{\prime} \text { disable }\right) \cdot(m \cdot S),
$$

i.e., $\{m\}=(m \triangleright$ disable $) \cdot(S \cup\{m\})$. We see that $m \triangleright$ disable should be a message that intersects the state with $\{m\}$. No $C_{1}^{\prime}$ message does this, but we can easily add one that does. This leads us to need more new messages, etc. Eventually we are led to define the messages of $C_{1}^{\prime}$ to be $M_{1}^{\prime}=\left\{\operatorname{disable}\left(S^{\prime}\right) \mid S^{\prime} \in \Sigma^{\prime}\right\}$, with disable $\left(S^{\prime}\right)$ acting as

$$
\operatorname{disable}\left(S^{\prime}\right) \cdot S=S \cap S^{\prime}
$$

The original operation disable is prepared as the message disable $(\emptyset)$. Then we can satisfy assumption (reordering) by setting

$$
m \triangleright \operatorname{disable}\left(S^{\prime}\right)=\operatorname{disable}\left(S^{\prime} \cup\{m\}\right) .
$$

Assumptions (action commutes) and (preserves authors) are trivially satisfied as well, where we again formally allow any replica to be an author of disable $\left(S^{\prime}\right)$. Thus we get a semidirect product CRDT

$$
C_{1}^{\prime} \rtimes_{D^{\prime}} C_{2}^{\prime}
$$

In this CRDT, the effect of an enable operation is to add itself to the internal state, making the externally visible value enabled. When a replica receives a message disable $(\emptyset)$ corresponding to a disable operation, it first acts by all concurrent enable messages. This leads to the message disable $\left(S^{\prime}\right)$, where $S^{\prime}$ is the set of all enable messages concurrent to the disable operation. That message is then applied to the state, thus removing any enable messages that were causally lesser than the disable operation. If this removes all enable messages, the internal state becomes $\emptyset$ and the externally visible value becomes disabled. Thus we have indeed implemented the enable-wins semantics as a semidirect product of commutative data types.

Remark 5.4. The internal state $S$ duplicates the role of the history set $H$ in the semidirect product $C_{1}^{\prime} \rtimes_{D^{\prime}} C_{2}^{\prime}$, except that it is trimmed to only contain relevant (not yet disabled) messages. Thus we can optimize the construction, without changing its semantics, by allowing $S$ to double as $H$.

We can likewise decompose the disable-wins flag, which is identical but with the roles of enable and disable switched.

\footnotetext{
${ }^{4}$ We need infinitely many of them so that it makes sense to talk about "the set of enable messages received so far" as a set containing one copy of enable for each time a replica issues an enable operation.
} 
Sets. Using a similar decomposition to the enable-wins flag, we can decompose two set CRDTs, the add-wins set and remove-wins set. These have a set as their externally visible state and operations $\operatorname{add}(a)$ and remove $(a)$ for $a$ in a universe of set elements, with the obvious sequential semantics. In the add-wins set, add $(a)$ operations win over concurrent remove $(a)$ operations, like in the enablewins flag. More precisely, letting $<$ be the causal order on messages, the value of an add-wins set after receiving messages $H$ is

$$
\left\{a \mid \exists(m=\operatorname{add}(a)) \in H . \forall\left(m^{\prime}=\operatorname{remove}(a)\right) \in H . m \nless m^{\prime}\right\} .
$$

In other words, the add-wins set functions like an enable-wins flag for each set element.

This leads us to decompose the add-wins set as the following semidirect product of commutative data types. Let $\Sigma$ be the state space whose states are sets $S$ of add $(a)$ messages, for $a$ in the universe of set elements. The externally visible value of a state $S \in \Sigma$ is $\{a \mid \exists(m=\operatorname{add}(a)) \in S\}$. Let $C_{2}$ be the commutative data type with state space $\Sigma$, operations add $(a)$ for $a$ in the universe, and infinitely many messages of the form add $(a)$ for each $a$, acting as $m \cdot \Sigma=\Sigma \cup\{m\}$. Let $C_{1}$ be the commutative data type with state space $\Sigma$, messages remove $(a)\left(S^{\prime}\right)$ for $S^{\prime}$ a set of $\operatorname{add}(a)$ messages, acting as

$$
\operatorname{remove}(a)\left(S^{\prime}\right) \cdot S=\{m \in S \mid m=\operatorname{add}(b), b \neq a\} \cup\left(S \cap S^{\prime}\right),
$$

and operations remove $(a)$ with corresponding prepared message remove $(a)(\emptyset)$. Define the semidirect product action $\triangleright$ by

$$
(m=\operatorname{add}(a)) \triangleright \operatorname{remove}(b)\left(S^{\prime}\right)= \begin{cases}\operatorname{remove}(b)\left(S^{\prime}\right) & \text { if } b \neq a \\ \operatorname{remove}(b)\left(S^{\prime} \cup\{m\}\right) & \text { if } b=a .\end{cases}
$$

Then it is easy to check assumptions (reordering), (action commutes), and (preserves authors), so we get a semidirect product CRDT $C_{1} \rtimes_{\triangleright} C_{2}$. This CRDT implements the add-wins semantics, as follows. The effect of an $\operatorname{add}(a)$ operation is to add itself to the internal state, making the externally visible value contain $a$. When a replica receives a message remove $(a)(\emptyset)$ corresponding to a remove $(a)$ operation, it first acts by all concurrent add messages. This leads to the message remove $(a)\left(S^{\prime}\right)$, where $S^{\prime}$ is the set of all add $(a)$ messages concurrent to the remove $(a)$ operation That message is then applied to the state, thus removing any add $(a)$ messages that were causally lesser than the remove $(a)$ operation. If this removes all add $(a)$ messages, the externally visible state no longer includes $a$.

The remove-wins set, in which the roles of add and remove are switched, can be decomposed analogously.

Reset-Wins Resettable CRDTs. Let $C$ be any CRDT, with state space $\Sigma$, message set $M$, and initial state $\sigma^{0}$. We wish to add a reset operation to $C$ which restores its state to $\sigma^{0}$. This is necessary for constructing dictionary CRDTs with values in $C$ [AntidoteDB 2019; Basho 2015]: removing a key triggers a reset on the corresponding value.

One possible concurrency semantics is reset-wins: any operation concurrent to a reset operation is ignored. When used in a dictionary CRDT, this leads to the remove-wins semantics, in which operations on a value have no effect if its key is concurrently removed [Preguiça 2018, §2.1.5].

To construct a reset-wins CRDT from $C$, we divide the operations into two sets, one containing the original operations on $C$ and one containing the single operation reset. For the reset-wins semantics, we want reset operations to come last in the arbitration order, so that they overwrite concurrent $C$ operations. This leads us to take the first component of the semidirect product to be $C$, and we take the second component to be the CRDT $C_{\text {reset }}$ with state space $\Sigma$ and a single operation reset acting as reset $\sigma=\sigma^{0}$. Note that $C_{\text {reset }}$ is a commutative data type. 
To define the action $\triangleright$ of $\{$ reset $\}$ on $M$, we expand $M$ to contain a message id acting as id $\cdot \sigma=\sigma$, and we set

$$
\text { reset } \triangleright m=\mathrm{id}
$$

for all $m \in M$. This satisfies assumption (reordering), since for all $m \in M$ and $\sigma \in \Sigma$,

$$
\text { reset } \cdot(m \cdot \sigma)=\sigma^{0}=\mathrm{id} \cdot(\text { reset } \cdot \sigma) \text {. }
$$

Assumptions (action commutes) and (preserves authors) are easily checked, where we formally allow any replica to be an author of id. Thus we get a semidirect product CRDT $C \rtimes_{\triangleright} C_{\text {reset }}$ implementing the reset-wins semantics.

Observed-Reset Resettable CRDTs. An alternative concurrency semantics for resets is observedreset: a reset only affects causally prior (i.e., observed) operations. The corresponding observedremove semantics is used in many dictionary CRDTs when a value's key is removed, including for most value types in Riak [Basho 2015], Antidote [AntidoteDB 2019], and the JSON CRDT of Kleppmann and Beresford [2016].

The observed-reset semantics corresponds to the arbitration order in which reset comes before concurrent operations of $C$, so that the concurrent operations are not overwritten by the reset. This is the opposite of the reset-wins arbitration order.

For the observed-reset semantics to make sense, we assume that for any execution of $C$ following Algorithm 1, any subset of the messages appearing in that execution can be applied to $\sigma^{0}$ in casual order, resulting in a defined state (not $\perp$ ). This ensures that if some messages are issued concurrently to a reset, they can still be applied to the reset state $\sigma^{0}$.

Our semidirect product construction is similar to the enable-wins flag and add-wins set. Let $C^{\prime}$ be the same as $C$ but with an extra component of the state storing a finite sequence $L$ of messages from $M$. When a message is applied to a state $(\sigma, L)$, it is appended to $L$ in addition to acting on $\sigma .^{5}$ Let $C_{\text {reset }}^{\prime}$ be a CRDT with the same state space as $C^{\prime}$ and with messages reset $\left(L^{\prime}\right)$ for $L^{\prime}$ a finite set of messages from $M$, acting as

$$
\operatorname{reset}\left(L^{\prime}\right) \cdot(\sigma, L)=\left(\left(L \cap L^{\prime}\right) \cdot \sigma^{0}, L \cap L^{\prime}\right)
$$

where $L \cap L^{\prime}$ denotes $L$ restricted to messages appearing in $L^{\prime}$, and $\left(L \cap L^{\prime}\right) \cdot \sigma^{0}$ denotes the result of applying the messages in $L \cap L^{\prime}$ to $\sigma^{0}$ in order. By assumption, $\left(L \cap L^{\prime}\right) \cdot \sigma^{0} \neq \perp$. Also, $C_{\text {reset }}^{\prime}$ has a single operation reset with corresponding prepared message reset $(\emptyset)$. Observe that $C_{\text {reset }}^{\prime}$ is a commutative data type.

Define the semidirect product action $\triangleright$ by

$$
m \triangleright \operatorname{reset}\left(L^{\prime}\right)=\operatorname{reset}\left(L^{\prime} \cup\{m\}\right),
$$

with $m$ appended to the end of the list. Then the semidirect product $C_{\text {reset }}^{\prime} \rtimes_{\triangleright} C^{\prime}$ has the operations of $C$ plus reset, and it implements the observed-reset semantics. Indeed, before a reset message is applied to the state, it is modified to ignore all concurrent messages that have already been applied to the state, and any concurrent messages that are later applied to the state are unaffected.

As with the enable-wins flag, we can optimize the construction by allowing the sequence $L$ appearing in the state to double as the semidirect product's history set.

\footnotetext{
${ }^{5}$ Technically, for $C^{\prime}$ to be a CRDT, we should treat $L$ as a causally ordered set of messages instead of a sequence, so that two replicas that add concurrent messages to $L$ in different orders end up in equivalent states.
} 


\section{GENERALITY}

\subsection{Interpretation as Operational Transformation}

Operational Transformation (OT) is an alternative technique for developing replicated data types that is often viewed as an opposing technique to CRDTs. OT predates CRDTs and is commonly used in applications, such as Google Docs. However, general OT is complicated. As a result, many OT algorithms have turned out to be incorrect [Imine et al. 2003]. CRDTs were introduced to avoid the complexities and errors of OT [Preguiça et al. 2009; Shapiro et al. 2011], by using extra metadata in states as well as prepared messages in place of operations, and by requiring commutativity of concurrent messages instead of transformation properties.

It is thus interesting that, even though the semidirect product is a CRDT construction that reproduces the semantics of many existing CRDTs, it can be viewed as a restricted kind of OT, as we now describe.

For our definition of OT, we use the framework of Ressel et al. [1996]. In this framework, to define an OT object with operations $M_{1} \cup M_{2}{ }^{6}$, we must define a transformation function

$$
t f_{1}:\left(M_{1} \cup M_{2}\right) \times\left(M_{1} \cup M_{2}\right) \rightarrow M_{1} \cup M_{2}
$$

satisfying Transformation Properties 1 and 2 in [Ressel et al. 1996] (copied in Theorem 6.2 below). When a replica receives an operation $m$ from another replica, it transforms $m$ by concurrent operations already in the history according to the adOPTed-algorithm [Ressel et al. 1996, Figure 8]. It then applies the resulting operation $m^{\prime}$ to its state and stores $m$.

Definition 6.1. Let $C_{1} \rtimes_{\triangleright} C_{2}$ be a semidirect product of CRDTs. Let $M_{1}$ be the messages of $C_{1}$ and $M_{2}$ those of $C_{2}$. We define the semidirect product transformation on $M_{1} \cup M_{2}$ by

$$
t f_{1}(m, l)= \begin{cases}l \triangleright m & \text { if } m \in M_{1} \text { and } l \in M_{2} \\ m & \text { otherwise. }\end{cases}
$$

This corresponds to the fact that when an operation $m \in M_{1}$ is applied after a concurrent operation $l \in M_{2}$, we apply $l \triangleright m$ instead of $m$.

Our CRDT construction essentially implements the operational transformation object corresponding to $t f_{1}$, except that we only store messages from $M_{2}$ in the history.

Theorem 6.2. The semidirect product transformation $t f_{1}$ satisfies Transformation Properties 1 and 2 of Ressel et al. [1996], i.e., ${ }^{7}$

1. For all potentially concurrent $l, m \in M_{1} \cup M_{2}$ and $\sigma \in \sum$ such that $l \cdot \sigma \neq \perp$ and $m \cdot \sigma \neq \perp$,

$$
l \cdot\left(t f_{1}(m, l) \cdot \sigma\right)=m \cdot\left(t f_{1}(l, m) \cdot \sigma\right)
$$

2. For all potentially concurrent $k, l, m \in M_{1} \cup M_{2}$,

$$
t f_{1}\left(t f_{1}(l, k), t f_{1}(m, k)\right)=t f_{1}\left(t f_{1}(l, m), t f_{1}(k, m)\right) .
$$

Thus by Ressel et al. [1996, Theorem 1], the corresponding OT object is eventually consistent. Furthermore, $C_{1} \rtimes_{\triangleright} C_{2}$ has the same semantics as this OT object.

Conversely, suppose we have an OT object whose operations can be partitioned into disjoint sets $O_{1}$ and $\mathrm{O}_{2}$, such that the transformation function $t f_{1}$ satisfies

$$
t f_{1}(o, p)=o \text { unless } o \in O_{1} \text { and } p \in O_{2} .
$$

\footnotetext{
${ }^{6}$ Unlike op-based CRDTs, OT objects typically do not differentiate between operations and messages, regarding them as the same.

${ }^{7}$ [Ressel et al. 1996] does not explicitly address the possibility that an operation may be undefined $(\perp)$ on a state, or that only certain combinations of operations can be concurrent. We slightly weaken the Transformation Properties to permit these possibilities.
} 
Then letting $C_{1}$ be the restriction of the OT object to $O_{1}$ operations (with $O_{1}$ also as the set of messages), $\mathrm{C}_{2}$ be its restriction to $\mathrm{O}_{2}$ operations, and $\triangleright: \mathrm{O}_{2} \times O_{1} \rightarrow O_{1}$ be given by

$$
o_{2} \triangleright o_{1}=t f_{1}\left(o_{1}, o_{2}\right) \text {, }
$$

we have that $C_{1}$ and $C_{2}$ are op-based CRDTs, and $C_{1} \rtimes_{\triangleright} C_{2}$ is a semidirect product CRDT with the same semantics as the OT object.

Proof. For the first statement, verifying the Transformation Properties is a simple case analysis. Transformation Property 1 holds when $l, m \in M_{1}$ because $C_{1}$ is a CRDT, and likewise for $l, m \in M_{2}$. The interesting case is when $l \in M_{1}$ and $m \in M_{2}$ or vice-versa, in which case it reduces to assumption (reordering). Transformation Property 2 is trivial in all cases except when $l \in M_{1}$ and $k, m \in M_{2}$, in which case it reduces to assumption (action commutes). (Assumption (preserves authors) only matters in that it ensures that "potential concurrency" behaves nicely under transformation.)

For the converse statement, $C_{1}$ and $C_{2}$ are easily CRDTs: if $o, o^{\prime} \in O_{1}$, then $t f_{1}\left(o, o^{\prime}\right)=o$ and $t f_{1}\left(o^{\prime}, o\right)=o^{\prime}$, so $o$ and $o^{\prime}$ commute by Transformation Property 1 , and similarly for $C_{2}$. The semidirect product assumption (reordering) holds by Transformation Property 1 again, assumption (action commutes) holds by Transformation Property 2, and (preserves authors) holds trivially as any message can have any author.

It remains to see, for both the CRDT-to-OT conversion and the converse conversion, that $C_{1} \rtimes_{\triangleright} C_{2}$ has the same semantics as the corresponding OT object. The adOPTed-algorithm transforms a received operation $o$ by all concurrent operations in the history, except that these operations must themselves be transformed by appropriate operations before they are used as transformers. While this can be complicated in general, in our case it does not matter: $\mathrm{M}_{2}$ messages (resp. $\mathrm{O}_{2}$ operations, for the OT-to-CRDT conversion) are always transformed trivially, and $M_{1}$ messages (resp. $O_{1}$ operations) never alter the target of their transformation. Hence the result of the adOPTed transformation applied to $m$ is always $m$ if $m \in M_{2}$, and it is the $m_{\text {act }}$ appearing on line 16 of Algorithm 2 if $m \in M_{1}$. Thus the semidirect product CRDT and the OT object end up applying the same message (resp. operation) to the state.

Remark 6.3. Although the semidirect product is a restricted kind of OT, it avoids OT's pitfalls. First, the restriction on $t f_{1}$ is severe: while general OT allows $t f_{1}$ to do anything, we require the result of applying $m$ followed by $t f_{1}(l, m)$ to always be equivalent to either $m$ followed by $l$ or $l$ followed by $m$, according to the arbitration order. This in turn simplifies Transformation Property 2 to assumption (action commutes), which is trivial to verify in all of our examples below, while for general OT algorithms it is often a source of incorrectness. Second, we allow the semidirect product's components to be full-fledged CRDTs, not just user operations acting on user-visible state. This allows us to use CRDTs to implement portions of the state that CRDTs are good at, like sequences, while using the semidirect product for conflicts that are best resolved using transformation. Indeed, it appears impossible to construct a sequence CRDT using semidirect products alone, since there is no clear arbitration order between conflicting insertions.

As a result, the semidirect product is more CRDT-like in character. Indeed, it reproduces the semantics of several existing CRDTs (Section 5). Additionally, we avoid the adOPTed-algorithm's need to store a multidimensional history of transformed operations computed using a doublyrecursive algorithm. Instead, our history is a subset of the messages actually sent by replicas, and these messages transform other messages directly instead of needing to be transformed recursively.

\subsection{Decomposing POLog CRDTs as Semidirect Products}

One existing general model for constructing op-based CRDTs is the POLog (partially ordered log) model of Baquero et al. [2017]. In that model, the externally visible state of a CRDT is defined as a 
function of the log of operations partially ordered by causality. We used this model implicitly when defining the semantics of the add-wins set in Section 5: letting $<$ be the causal order on messages, the value of an add-wins set after receiving messages $H$ is

$$
f_{\text {aw-set }}(H, \prec):=\left\{a \mid \exists(m=\operatorname{add}(a)) \in H . \forall\left(m^{\prime}=\operatorname{remove}(a)\right) \in H . m \nless m^{\prime}\right\} .
$$

The POLog model advocates using $f_{\text {aw-set }}$ directly to implement an add-wins set, by applying it to the current message history each time a user queries the set's state. This is in contrast to traditional op-based CRDT designs, which typically store a metadata-enhanced version of the original data type's state instead of the full message history.

Given a POLog CRDT, it is interesting to ask whether the CRDT can be decomposed as a semidirect product of simpler CRDTs, by which we mean CRDTs with fewer operations. Ideally, we would like to repeat this decomposition until we get an iterated semidirect product of commutative data types. We can then build up an alternate construction of the original CRDT using these semidirect products. This can clarify the semantics and suggest a more efficient implementation of the original CRDT, by reasoning about conflicts between concurrent messages in a restricted, uniform way instead of allowing the full power of a POLog function.

The following proposition gives a general condition under which a POLog CRDT can be decomposed as the semidirect product of two simpler CRDTs.

Proposition 6.4. Let $f$ be a function defining a POLog CRDTC with operation set $O_{1} \cup O_{2}$, i.e., $f$ is a function mapping a partially ordered log of operations in $O_{1} \cup O_{2}$ to an externally visible state. Suppose that $f(L)=f\left(L^{\prime}\right)$ whenever $L$ and $L^{\prime}$ are partially ordered logs differing only in that, for some message $m_{1}$ corresponding to an $O_{1}$ operation and some message $m_{2}$ corresponding to an $\mathrm{O}_{2}$ operation, $m_{1} \prec m_{2}$ in the partial order of $L$ while $m_{1}$ is concurrent to $m_{2}$ in the partial order of $L^{\prime}$. Then $C$ has the same externally visible semantics as some semidirect product of $C_{1}$ and $C_{2}$, where $C_{1}$ (resp. $C_{2}$ ) is a CRDT with the same externally visible semantics as the restriction of $C$ to $O_{1}$ operations (resp. $\mathrm{O}_{2}$ operations).

Proof Sketch. To define $C_{1}$ and $C_{2}$, we start with the POLog CRDTs derived from the restriction of $f$ to $O_{1}$ operations (resp., $O_{2}$ operations), modified so that their partially ordered logs may each contain both $O_{1}$ and $O_{2}$ operations. We then modify the messages of $C_{1}$ so that in addition to an $O_{1}$ operation and a timestamp, they contain a set $S$ of $C_{2}$ messages. We define the semidirect product action by

$$
m_{2} \triangleright\left(m_{1}, S\right)=\left(m_{1}, S \cup\left\{m_{2}\right\}\right) .
$$

Finally, we define the effect of $\left(m_{1}, S\right)$ to be to add $m_{1}$ to the log with partial order relations: $m_{1}^{\prime} \prec m_{1}$ for all $m_{1}^{\prime}$ corresponding to $O_{1}$ operations with lower timestamps; $m_{2} \prec m_{1}$ for all $m_{2}$ corresponding to $\mathrm{O}_{2}$ operations that are not in $S$; and any additional relations required by transitivity (so that $<$ remains a partial order). Note that the timestamps on $O_{1}$ operations are unrelated to those on $\mathrm{O}_{2}$ operations, so that $\triangleright$ is the only way we can reason about concurrent $O_{1}$ and $O_{2}$ operations.

After performing an execution on both $C$ and $C_{1} \rtimes_{\triangleright} C_{2}$, the resulting partially ordered logs $L$ and $L^{\rtimes}$ differ only in that, for some messages $m_{1}, m_{2} \in L$ corresponding to an $O_{1}$ operation and an $O_{2}$ operation, respectively, $m_{1} \prec m_{2}$ in $L$ but $m_{1}$ is concurrent to $m_{2}$ in $L^{\rtimes}$. Thus by hypothesis, $f(L)=f\left(L^{\rtimes}\right)$.

Of the non-commutative POLog CRDTs described by Baquero et al. [2017], only the multi-value register cannot be decomposed using this proposition, while the enable-wins flag, disable-wins flag, add-wins set, and remove-wins set decompose into semidirect products of commutative data types, as described in Section 5. 


\section{OPTIMIZATIONS}

As defined above, a state of $C_{1} \rtimes_{\triangleright} C_{2}$ includes the set $H$ of all $M_{2}$ messages that have already been applied. This set can grow without bound, potentially making the state large and affecting the performance of an implementation. We now discuss two optimizations that can reduce this state size.

\subsection{Causal Stability}

In a state $(\sigma, t, H)$ of $C_{1} \rtimes_{\triangleright} C_{2}$, observe that a pair $\left(m, t^{\prime}\right) \in H$ only matters when we apply a message concurrent to it. Thus once $\left(m, t^{\prime}\right)$ becomes causally stable [Baquero et al. 2017, §5.2], meaning that all future inputs $\left(l, s^{\prime}\right)$ to effect ${ }_{\rtimes}$ will be causally greater than $\left(m, t^{\prime}\right)$, we can discard $\left(m, t^{\prime}\right)$ from $H$ without changing the externally visible behavior of $C_{1} \rtimes_{\triangleright} C_{2}$.

\subsection{Compressing the History}

In some cases, instead of storing the history of $M_{2}$ messages in our state as a set $H$, we can store a single $M_{2}$ message representing the composition of all of these messages. Specifically, assume:

- $M_{2}$ is closed under composition, in the sense that for all $m_{2}, m_{2}^{\prime} \in M_{2}$, there exists a message $m_{2} \circ m_{2}^{\prime} \in M_{2}$ such that for all $\sigma \in \Sigma,\left(m_{2} \circ m_{2}^{\prime}\right) \cdot \sigma=m_{2} \cdot\left(m_{2}^{\prime} \cdot \sigma\right)$, and for all $m_{1} \in M_{1}$, $\left(m_{2} \circ m_{2}^{\prime}\right) \triangleright m_{1}=m_{2} \triangleright\left(m_{2}^{\prime} \triangleright m_{1}\right)$.

- $M_{2}$ messages commute (not just when they are concurrent), i.e., for all $m_{2}, m_{2}^{\prime} \in M_{2}, m_{2} \circ m_{2}^{\prime}=$ $m_{2}^{\prime} \circ m_{2}$.

- For all $m_{2} \in M_{2}$, the function $m_{2}^{\prime} \mapsto m_{2} \circ m_{2}^{\prime}$ is injective. We let $m_{2}^{-1}$ be a formal symbol acting as the corresponding inverse partial function, i.e., $m_{2}^{-1} \circ\left(m_{2} \circ m_{2}^{\prime}\right):=m_{2}^{\prime}$.

Example 7.1. The example of Section 3.1 satisfies these assumptions if we exclude mult $(0)$, with $\operatorname{mult}(n) \circ \operatorname{mult}\left(n^{\prime}\right)=\operatorname{mult}\left(n n^{\prime}\right)$ and with $\operatorname{mult}(n)^{-1} \circ \operatorname{mult}(m)=\operatorname{mult}(m / n)$ when $n$ divides $m$.

Remark 7.2. In the language of abstract algebra, these assumptions hold whenever $M_{2}$ is an abelian group with binary operation $\circ$, and the functions $\cdot: M_{2} \times \Sigma \rightarrow \Sigma, \triangleright: M_{2} \times M_{1} \rightarrow M_{1}$ are both group actions. Indeed, this algebraic statement is equivalent except that it additionally requires that the formal symbols $m_{2}^{-1}$ are literal messages in $M_{2}$.

To accommodate the initial state, we formally add the identity function id to $M_{2}$.

Definition 7.3. The compressed semidirect product of $C_{1}$ and $C_{2}$ is the op-based CRDT $C_{1} \rtimes_{\triangleright}^{\text {comp }} C_{2}=$ $\left(\Sigma \times M_{2},\left(\sigma^{0}\right.\right.$, id $)$, prepare comp , effect comp $_{\text {, eval }}$ comp $)$ with components defined in Algorithm 3 .

One can show that $C_{1} \rtimes_{\triangleright}^{\text {comp }} C_{2}$ satisfies the CRDT properties using a proof similar to Theorem 3.4. Informally, instead of storing the history $H$, we store the composition $h$ of all messages in $H$. When applying an $M_{1}$ message $\left(m, h^{\prime}\right)$ to a state $(\sigma, h), h^{\prime}$ is the composition of all $M_{2}$ messages causally prior to $\left(m, h^{\prime}\right)$, while $h$ is the composition of all $M_{2}$ messages applied to the state. Thus $\left(h^{\prime}\right)^{-1} \circ h$ is the composition of all $M_{2}$ messages applied to the state that are concurrent to $\left(m, h^{\prime}\right)$ instead of causally prior to it, so that $m_{\text {act }}$ is the same as it would be in $C_{1} \rtimes_{\triangleright} C_{2}$.

Example 7.4. We can use the compressed semidirect product for the following examples above:

- The sequence with reverse operation, since reverse is closed under composition (after adding an identity operation id), commutative, and invertible. The resulting CRDT is essentially equivalent to treating the possibly-reversed sequence as a view of an ordinary sequence CRDT, with reverse operations toggling the view, and with user inputs reversed whenever they are performed on a reversed view. 


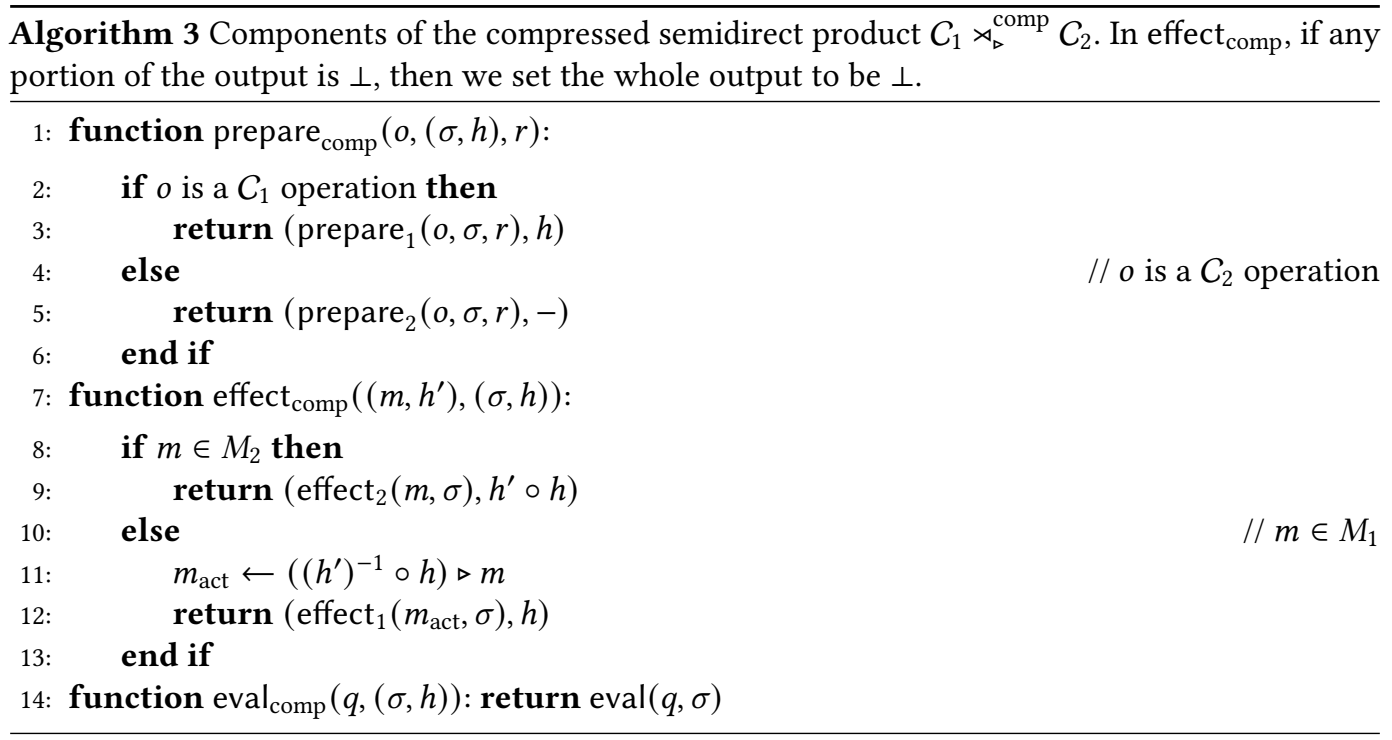

- The semiring CRDT, whenever the semiring $(S, \oplus, \otimes)$ is such that for all $s \in S$, the function $t \mapsto s \otimes t$ is injective. This includes the example of Section 3.1 if we exclude mult( 0 ) operations, which corresponds to the semiring $(\mathbb{Z},+, \times)$.

It also includes the semiring $(\mathbb{N}, \min ,+)$. This can be used as a form of resettable counter, with $\min (0)$ behaving as a reset-to- 0 operation. It is practically interesting because we can implement this CRDT with constant-sized state, in contrast to existing resettable counter designs. However, it does not have either of the typical resettable CRDT semantics described in Section 5 (reset-wins or observed-reset). It is similar to observed-reset, but with the following anomaly:

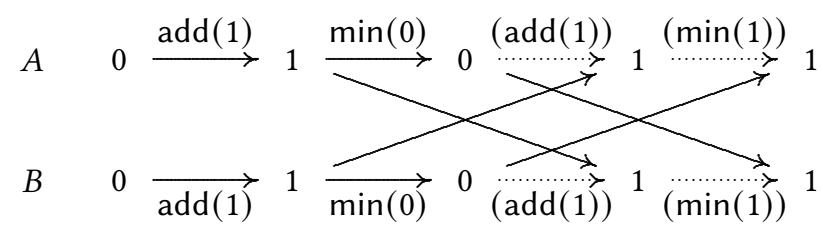

Here replicas $A$ and $B$ both increment the counter and then reset it, but the add(1) operations transform the concurrent $\min (0)$ operations into $\min (1)$ operations, giving a final state of 1 . Meanwhile, the observed-reset semantics would give a final state of 0 .

Nonetheless, this resettable counter may still be useful, since the large state size of true observed-reset resettable counters [Younes et al. 2017] has led some to adopt alternative semantics in return for smaller state [Baquero et al. 2016; Basho 2015].

\section{RELATED WORK}

Several works describe general techniques for constructing replicated data types. Leijnse et al. [2019] discuss patterns in existing op-based CRDTs, such as constructions of set CRDTs from flag CRDTs. The patterns they identify are orthogonal to the semidirect product.

Baquero et al. [2015] give general techniques for composing state-based CRDTs, the other kind of CRDTs besides op-based, using lattice merge functions. Similar techniques are used by the Bloom ${ }^{L}$ distributed programming model [Conway et al. 2012] and the LVars parallel programming model 
[Kuper and Newton 2013]. While those works use lattice theory to compose state-based CRDTs, we use an idea from abstract algebra to compose op-based CRDTs. Also, those works (especially Conway et al. [2012] and Kuper and Newton [2013]) focus on composition in the sense of composite data types (e.g., tuples and dictionaries), while we focus on composing different operations acting on the same base data type.

Mergeable Replicated Data Types (MRDTs), defined by Kaki et al. [2019] and built on top of Irmin [MirageOS Project 2020], are branch-and-merge based replicated data types that use a three-way merge function for sets to define MRDTs for various data types automatically. Like us, Kaki et al. define a replicated integer register supporting addition and multiplication operations, but with different semantics: a multiplication is treated as its equivalent addition. Our construction is less automatic but more flexible: MRDTs are only defined for data types built as views of relations on sets, and they give at most one semantics for a given data type, excluding examples like a remove-wins set or a reset-wins resettable type. In contrast, our examples demonstrate the semidrect product's wide applicability. Also, MRDT's branch-and-merge system model more closely resembles state-based CRDTs, in contrast to our use of op-based CRDTs.

A particular kind of arbitration between concurrent CRDT operations appears in work on tunable CRDTs by Rijo [2018] and the concept of "cast-off updates" in a survey by Preguiça [2018]. Specifically, they consider operations that become irrelevant due to other operations, such as removes cancelled by adds in an add-wins set. Rijo gives a construction based on generic arbitration rules, in which operations of one type can cancel those of another depending on their causal relationship.

As described in Section 6.1, the semidirect product can be viewed as a restricted kind of operational transformation (OT). We avoid the complexity of general OT approaches by effectively only allowing the transformation function to reorder operations, not arbitrarily transform them, and only in the specific case of a $C_{2}$ operation followed by a $C_{1}$ operation. Also, we allow that the state is a CRDT state and the operations being transformed are CRDT messages, not just user operations acting on user-visible state, so that the components of a semidirect product may continue using CRDT techniques.

Lasp [Meiklejohn and Van Roy 2015] is an Erlang programming model that allows one to create views of a given CRDT, such as a functionally mapped view of a set, which update in an eventually consistent way. We focus on adding in-place, mutating operations to CRDTs, as opposed to creating immutable views. Indeed, several of our examples, such as the integer register with addition and multiplication operations and the dictionary with a map operation, were motivated by the goal of developing CRDTs that support these operations in-place instead of as views.

OpSets [Kleppmann et al. 2018] and SECROs [De Porre et al. 2019] both convert generic data types into replicated data types by sorting operations into an eventually consistent total order compatible with the causal order. While we aim to sort operations so that they respect both the causal order and the arbitration order, this is not always possible (see Section 3.1), so we instead use the transformation $\triangleright$ to approximate the desired order. As a result, the semidirect product gives semantics more typical of CRDTs than OpSets or SECROs: conflicts between concurrent operations are resolved uniformly according to an arbitration order chosen at design time, instead of according to an arbitrary total order at run time. Also, through use of the transformation $\triangleright$, we avoid the need to re-order and re-apply operations that are received out-of-order.

\section{CONCLUSION}

We introduced the semidirect product of op-based CRDTs. This construction combines the operations of two CRDTs while handling concurrency conflicts between them in a uniform way. Specifically, it implements an arbitration order on concurrent messages using a restricted kind of

Proc. ACM Program. Lang., Vol. 4, No. ICFP, Article 94. Publication date: August 2020. 
operational transformation. We constructed novel CRDTs through composition, and our examples also showed that several existing CRDTs can be decomposed as semidirect products of simpler CRDTs.

For future work, we plan to investigate iterated semidirect products. In particular, it would be interesting to see whether complicated CRDTs can be decomposed as iterated semidirect products of commutative data types, thus completely handling concurrency conflicts through the semidirect product. We will also pursue implementations of the novel CRDTs described above.

\section{ACKNOWLEDGMENTS}

We thank Carlos Baquero for feedback on an early draft of this work. We also thank the anonymous reviewers for their many helpful comments.

\section{A ALGEBRAIC MOTIVATION}

The semidirect product of CRDTs is inspired by the semidirect product of groups, which we now describe.

In abstract algebra, a group is a set $G$ together with a binary operation $\bullet: G \times G \rightarrow G$ such that:

- $\bullet$ is associative: $g \bullet(h \bullet k)=(g \bullet h) \bullet k$

- There is an identity $1_{G} \in G$ satisfying $1_{G} \bullet g=g \bullet 1_{G}=g$

- Each $g \in G$ has an inverse $g^{-1}$ such that $g \bullet g^{-1}=g^{-1} \bullet g=1_{G}$.

Let $\left(G_{1}, \bullet_{1}\right)$ and $\left(G_{2}, \bullet_{2}\right)$ be groups. Suppose we have an action $\triangleright: G_{2} \times G_{1} \rightarrow G_{1}$ satisfying:

$-g_{2} \triangleright\left(g_{1} \bullet{ }_{1} g_{1}^{\prime}\right)=\left(g_{2} \triangleright g_{1}\right) \bullet{ }_{1}\left(g_{2} \triangleright g_{1}^{\prime}\right)$

$-\left(g_{2} \bullet \bullet_{2} g_{2}^{\prime}\right) \triangleright g_{1}=g_{2} \triangleright\left(g_{2}^{\prime} \triangleright g_{1}\right)$

$-1_{G_{2}} \triangleright g_{1}=g_{1}$

- For each $g_{2}, g_{1} \mapsto g_{2} \triangleright g_{1}$ is an invertible function.

Then the semidirect product of $G_{1}$ and $G_{2}$ with respect to $\triangleright$ [Dummit and Foote 2004, §5.5] is the group $G_{1} \rtimes_{\triangleright} G_{2}$ with underlying set $G_{1} \times G_{2}$ and binary operation

$$
\left(g_{1}, g_{2}\right) \bullet \bullet_{\rtimes}\left(g_{1}^{\prime}, g_{2}^{\prime}\right):=\left(g_{1} \bullet_{1}\left(g_{2} \triangleright g_{1}^{\prime}\right), g_{2} \bullet_{2} g_{2}^{\prime}\right) .
$$

We can think of $G_{1}$ and $G_{2}$ as subgroups of $G_{1} \rtimes_{\triangleright} G_{2}$ (i.e., subsets that are groups) via the maps $g_{1} \mapsto\left(g_{1}, 1_{G_{2}}\right), g_{2} \mapsto\left(1_{G_{1}}, g_{2}\right)$. If we put the elements corresponding to $g_{1}$ and $g_{2}$ in the "wrong" order $\left(g_{2}\right.$ then $\left.g_{1}\right)$, they get rearranged as

$$
\left(1_{G_{1}}, g_{2}\right) \bullet_{\rtimes}\left(g_{1}, 1_{G_{2}}\right)=\left(g_{2} \triangleright g_{1}, g_{2}\right)=\left(g_{2} \triangleright g_{1}, 1_{G_{2}}\right) \bullet_{\rtimes}\left(1_{G_{1}}, g_{2}\right),
$$

i.e., as $\left(g_{2} \triangleright g_{1}\right.$ then $\left.g_{2}\right)$. This inspired the semidirect product CRDT's use of the equivalence between $\left(m_{2} \triangleright m_{1}\right) \circ m_{2}$ and $m_{2} \circ m_{1}$, for $m_{1}$ a $C_{1}$ message and $m_{2}$ a $C_{2}$ message, as a way to reorder messages so that $C_{1}$ messages effectively come before $C_{2}$ messages.

\section{REFERENCES}

AntidoteDB. 2019. Datatypes in Antidote. https://antidotedb.gitbook.io/documentation/architecture/datatypes. Carlos Baquero, Paulo Sérgio Almeida, Alcino Cunha, and Carla Ferreira. 2015. Composition of State-based CRDTs. (05/25 2015).

Carlos Baquero, Paulo Sérgio Almeida, and Carl Lerche. 2016. The Problem with Embedded CRDT Counters and a Solution. In Proceedings of the 2nd Workshop on the Principles and Practice of Consistency for Distributed Data (London, United Kingdom) (PaPoC '16). Association for Computing Machinery, New York, NY, USA, Article 10, 3 pages. https: //doi.org/10.1145/2911151.2911159

Carlos Baquero, Paulo Sérgio Almeida, and Ali Shoker. 2017. Pure Operation-Based Replicated Data Types. CoRR abs/1710.04469 (2017). arXiv:1710.04469 http://arxiv.org/abs/1710.04469

Basho. 2015. Riak datatypes. http://github.com/basho. 
Neil Conway, William R. Marczak, Peter Alvaro, Joseph M. Hellerstein, and David Maier. 2012. Logic and Lattices for Distributed Programming. In Proceedings of the Third ACM Symposium on Cloud Computing (San Jose, California) (SoCC '12). Association for Computing Machinery, New York, NY, USA, Article 1, 14 pages. https://doi.org/10.1145/2391229. 2391230

Kevin De Porre, Florian Myter, Christophe De Troyer, Christophe Scholliers, Wolfgang De Meuter, and Elisa Gonzalez Boix. 2019. A Generic Replicated Data Type for Strong Eventual Consistency. In Proceedings of the 6th Workshop on Principles and Practice of Consistency for Distributed Data (Dresden, Germany) (PaPoC '19). Association for Computing Machinery, New York, NY, USA, Article 8, 3 pages. https://doi.org/10.1145/3301419.3323974

Xavier Défago, André Schiper, and Péter Urbán. 2004. Total Order Broadcast and Multicast Algorithms: Taxonomy and Survey. ACM Comput. Surv. 36, 4 (Dec. 2004), 372-421. https://doi.org/10.1145/1041680.1041682

David S. Dummit and Richard M. Foote. 2004. Abstract Algebra (3rd ed.). Wiley.

Colin J. Fidge. 1988. Timestamps in message-passing systems that preserve the partial ordering. Proceedings of the 11th Australian Computer Science Conference 10, 1 (1988), 56-66.

Abdessamad Imine, Pascal Molli, Gérald Oster, and Michaël Rusinowitch. 2003. Proving Correctness of Transformation Functions in Real-Time Groupware. In ECSCW 2003, Kari Kuutti, Eija Helena Karsten, Geraldine Fitzpatrick, Paul Dourish, and Kjeld Schmidt (Eds.). Springer Netherlands, Dordrecht, 277-293.

Gowtham Kaki, Swarn Priya, KC Sivaramakrishnan, and Suresh Jagannathan. 2019. Mergeable Replicated Data Types. Proc. ACM Program. Lang. 3, OOPSLA, Article 154 (Oct. 2019), 29 pages. https://doi.org/10.1145/3360580

Martin Kleppmann and Alastair R. Beresford. 2016. A Conflict-Free Replicated JSON Datatype. CoRR abs/1608.03960 (2016) arXiv:1608.03960 http://arxiv.org/abs/1608.03960

Martin Kleppmann, Victor B. F. Gomes, Dominic P. Mulligan, and Alastair R. Beresford. 2018. OpSets: Sequential Specifications for Replicated Datatypes (Extended Version). CoRR abs/1805.04263 (2018). arXiv:1805.04263 http://arxiv.org/abs/1805. 04263

Lindsey Kuper and Ryan R. Newton. 2013. LVars: Lattice-Based Data Structures for Deterministic Parallelism. In Proceedings of the 2nd ACM SIGPLAN Workshop on Functional High-Performance Computing (Boston, Massachusetts, USA) (FHPC '13). Association for Computing Machinery, New York, NY, USA, 71-84. https://doi.org/10.1145/2502323.2502326

Adriaan Leijnse, Paulo Sérgio Almeida, and Carlos Baquero. 2019. Higher-Order Patterns in Replicated Data Types. In Proceedings of the 6th Workshop on Principles and Practice of Consistency for Distributed Data (Dresden, Germany) (PaPoC '19). Association for Computing Machinery, New York, NY, USA, Article 5, 6 pages. https://doi.org/10.1145/3301419. 3323971

Friedemann Mattern. 1989. Virtual Time and Global States of Distributed Systems. In Parallel and Distributed Algorithms. North-Holland, 215-226.

Christopher Meiklejohn and Peter Van Roy. 2015. Lasp: A Language for Distributed, Coordination-Free Programming. In Proceedings of the 17th International Symposium on Principles and Practice of Declarative Programming (Siena, Italy) (PPDP '15). Association for Computing Machinery, New York, NY, USA, 184-195. https://doi.org/10.1145/2790449.2790525

MirageOS Project. 2020. Irmin. https://irmin.org/.

Nuno Preguiça, Carlos Baquero, and Marc Shapiro. 2018. Conflict-Free Replicated Data Types CRDTs. Springer International Publishing, Cham, 1-10. https://doi.org/10.1007/978-3-319-63962-8_185-1

Nuno M. Preguiça. 2018. Conflict-free Replicated Data Types: An Overview. CoRR abs/1806.10254 (2018). arXiv:1806.10254 http://arxiv.org/abs/1806.10254

Nuno Preguiça, Joan M. Marquès, Marc Shapiro, and Mihai Leţia. 2009. A Commutative Replicated Data Type for Cooperative Editing. In 2009 29th IEEE International Conference on Distributed Computing Systems. 395-403. https://doi.org/10.1109/ ICDCS.2009.20

Matthias Ressel, Doris Nitsche-Ruhland, and Rul Gunzenhäuser. 1996. An Integrating, Transformation-Oriented Approach to Concurrency Control and Undo in Group Editors. In Proceedings of the 1996 ACM Conference on Computer Supported Cooperative Work (Boston, Massachusetts, USA) (CSCW'96). Association for Computing Machinery, New York, NY, USA, 288-297. https://doi.org/10.1145/240080.240305

André dos Reis Martins Rijo. 2018. Building Tunable CRDTs. Master's thesis. Universidade NOVA de Lisboa. http: //hdl.handle.net/10362/55171.

Azriel Rosenfeld. 1968. An introduction to algebraic structures. Holden-Day, San Francisco.

Marc Shapiro, Nuno Preguiça, Carlos Baquero, and Marek Zawirski. 2011. A comprehensive study of Convergent and Commutative Replicated Data Types. Research Report RR-7506. Inria - Centre Paris-Rocquencourt ; INRIA. 50 pages. https://hal.inria.fr/inria-00555588

Matthew Weidner, Heather Miller, and Christopher Meiklejohn. 2020. Composing and Decomposing Op-Based CRDTs with Semidirect Products: (Summary). In Proceedings of the 7th Workshop on Principles and Practice of Consistency for Distributed Data (Heraklion, Greece) (PaPoC '20). Association for Computing Machinery, New York, NY, USA, Article 14, 2 pages. https://doi.org/10.1145/3380787.3393687 
Georges Younes, Paulo Sérgio Almeida, and Carlos Baquero. 2017. Compact Resettable Counters Through Causal Stability. In Proceedings of the 3rd International Workshop on Principles and Practice of Consistency for Distributed Data (Belgrade, Serbia) (PaPoC '17). ACM, New York, NY, USA, Article 2, 3 pages. https://doi.org/10.1145/3064889.3064892 